\title{
Contrasting invertebrate immune defense behaviors caused by a single gene, the Caenorhabditis elegans neuropeptide receptor gene $n p r-1$
}

Rania Nakad ${ }^{1}{ }^{1}$, L. Basten Snoek ${ }^{3}$, Wentao Yang ${ }^{1}$, Sunna Ellendt ${ }^{1}$, Franziska Schneider ${ }^{1}$, Timm G. Mohr ${ }^{1}$, Lone Rösingh ${ }^{1}$, Anna C. Masche ${ }^{1}$, Philip C. Rosenstiel ${ }^{4}$, Katja Dierking ${ }^{1}$, Jan E. Kammenga ${ }^{3}$

and Hinrich Schulenburg ${ }^{1 *}$

\begin{abstract}
Background: The invertebrate immune system comprises physiological mechanisms, physical barriers and also behavioral responses. It is generally related to the vertebrate innate immune system and widely believed to provide nonspecific defense against pathogens, whereby the response to different pathogen types is usually mediated by distinct signalling cascades. Recent work suggests that invertebrate immune defense can be more specific at least at the phenotypic level. The underlying genetic mechanisms are as yet poorly understood.

Results: We demonstrate in the model invertebrate Caenorhabditis elegans that a single gene, a homolog of the mammalian neuropeptide $Y$ receptor gene, $n p r-1$, mediates contrasting defense phenotypes towards two distinct pathogens, the Gram-positive Bacillus thuringiensis and the Gram-negative Pseudomonas aeruginosa. Our findings are based on combining quantitative trait loci (QTLS) analysis with functional genetic analysis and RNAseq-based transcriptomics. The QTL analysis focused on behavioral immune defense against $B$. thuringiensis, using recombinant inbred lines (RILs) and introgression lines (ILS). It revealed several defense QTLs, including one on chromosome X comprising the npr-1 gene. The wildtype N2 allele for the latter QTL was associated with reduced defense against $B$. thuringiensis and thus produced an opposite phenotype to that previously reported for the $\mathrm{N} 2 \mathrm{npr}-1$ allele against $P$. aeruginosa. Analysis of npr-1 mutants confirmed these contrasting immune phenotypes for both avoidance behavior and nematode survival. Subsequent transcriptional profiling of C. elegans wildtype and npr-1 mutant suggested that npr-1 mediates defense against both pathogens through p38 MAPK signaling, insulin-like signaling, and C-type lectins. Importantly, increased defense towards $P$. aeruginosa seems to be additionally influenced through the induction of oxidative stress genes and activation of GATA transcription factors, while the repression of oxidative stress genes combined with activation of Ebox transcription factors appears to enhance susceptibility to B. thuringiensis.

Conclusions: Our findings highlight the role of a single gene, npr-1, in fine-tuning nematode immune defense, showing the ability of the invertebrate immune system to produce highly specialized and potentially opposing immune responses via single regulatory genes.
\end{abstract}

Keywords: Caenorhabditis elegans, Pathogen avoidance behavior, Innate immunity, Immune specificity, QTL analysis

\footnotetext{
* Correspondence: hschulenburg@zoologie.uni-kiel.de

'Department of Evolutionary Ecology and Genetics, Zoological Institute,

University of Kiel, 24098 Kiel, Germany

Full list of author information is available at the end of the article
} 


\section{Background}

In contrast to higher vertebrates, which have adaptive immune response systems, invertebrates exclusively rely on the innate immune system (the immune system is here defined sensu lato as the organism's defense against infection, including avoidance behavior, physical barriers, and physiological processes). For a long time it was assumed that only the adaptive system is capable of mounting highly specific defense responses. However, evidence is accumulating that invertebrates have surprisingly complex immune systems that in theory may have the potential to produce similar specificities [1-3]. Yet, to date, we possess only little information on the genetic and molecular mechanisms underlying such specificities. First insights into these mechanisms were previously obtained for the model nematode C. elegans, an important invertebrate system for studying immune defense [2, 4, 5]. For example, loss of function of the prolylhydroxylase encoding gene egl9 enhances susceptibility to Staphylococcus aureus [6] but resistance to Pseudomonas aeruginosa (PA01) [7] and Bacillus thuringiensis toxins [8]. Similarly, a loss of function of the Toll-like receptor gene tol-1 increases susceptibility to Salmonella enterica but resistance to Enterococcus faecalis [9], even though the general importance of tol-1 in worm immunity is unclear $[5,10]$.

Such specificities may not only be expressed by the nematode's physiological immune system, but could also be expected for behavioral defenses. Such behaviors are a central component of immune defense sensu lato - next to protective barriers and physiological processes - and are likely to represent a highly economic immune defense strategy because they simultaneously reduce pathogen contact, and thus the risk of tissue damage, and also the necessity to activate the energetically costly physiological and cellular response [11]. C. elegans colonizes microberich habitats in nature where it feeds on bacteria and yeasts [12-15]. Since these habitats also contain many pathogenic microorganisms, C. elegans has evolved distinct types of behavioral responses including physical avoidance, associative learning and reduced oral uptake of pathogens $[4,16-22]$.

Previous studies revealed the presence of substantial genetic variation among wild isolates of C. elegans in their behavioral response towards different pathogens $[17,19,23-27]$. In one case, namely the defense response against the Gram-negative bacterium Pseudomonas aeruginosa, this variation could be linked to the polymorphic neuropeptide receptor $n p r-1$ locus on the X chromosome. The gene $n p r-1$ was proposed to regulate C. elegans' immunity against PA14 either through controlling the aerotaxis response [17], or through controlling both aerotaxis response and physiological immune defense [18]. npr-1 is a homolog of the mammalian neuropeptide $\mathrm{Y}$ receptor gene and it is found in two different isoforms in C. elegans that result from a single amino acid change at position 215 (valine in isoform $215 \mathrm{~V}$; phenylalanine in isoform $215 \mathrm{~F}$ ) [28]. These isoforms do not only influence pathogen defense but also foraging behavior in response to oxygen concentrations [28, 29] and leaving behavior from lawns with the laboratory food bacterium Escherichia coli [30, 31].

The apparent complexity of the $C$. elegans defense against pathogens $[1-3,5]$ raises the question whether single pathways or genes can also fine-tune the behavioral defense response towards specific pathogens. To address this question we studied the genetic architecture of behavioral immune defense of $C$. elegans towards the Grampositive pathogen Bacillus thuringiensis. This pathogen is likely to coexist with $C$. elegans in nature [15]. Some strains are nematocidal, whereby the host is infected by the oral uptake of spore-toxin mixtures. Infection of the gut is followed by toxin-mediated cellular damage of the intestinal epidermis, germination of spores and subsequent proliferation of vegetative cells, including expression of various virulence factors, ultimately resulting in nematode death [32-36]. Nematocidal B. thuringiensis induces pronounced behavioral responses in C. elegans [21, 23, 37, 38].

Here we explored genetic variation in $C$. elegans and used quantitative trait locus (QTL) analysis to characterize the genetic basis of behavioral immune defense against two pathogenic B. thuringiensis strains, whereby one strains (BT B-18679) is known to be more pathogenic than the other (BT B-18247) [39, 40]. Our QTL analysis was based on a panel of 200 recombinant inbred lines (RILs) and 90 introgression lines (ILs), derived from a cross between the C. elegans strains N2 and CB4856 [41, 42]. Our QTL analysis identified $n p r-1$ as one of the candidate genes, though with an opposite effect on avoidance behavior to that previously reported towards $P$. aeruginosa [17, 18]. Therefore, we further characterized the function of the $n p r-1$ gene in producing contrasting pathogen defense responses. Using $n p r-1$ mutants, we assessed the influence of the gene on both avoidance behavior and survival towards the two pathogen species, B. thuringiensis and $P$. aeruginosa. Moreover, we used RNAseq to identify differences in the pathogen-dependent transcription of npr-1 down-stream targets. The functional importance of such differences was assessed through enrichment analysis of gene ontology (GO) categories, customized nematode-specific gene sets, which we collated from previous gene expression analyses, and transcription factor binding motifs.

\section{Results and discussion}

Two C. elegans wild-type strains differ in bacterial lawn leaving behavior

The standard laboratory strain N2 and the Hawaiian strain CB4856 showed significant variation in lawn-leaving behavior towards nematocidal B. thuringiensis strains (B- 
18679 \& B-18247) and non-nematocidal strains (DSM350 \& E. coli OP50). Lawn leaving served as a proxy for behavioral defense and was based on an assay (Additional file 1) related to those previously used to characterize $C$. elegans avoidance behavior [10, 16, 19, 21, 31, 37], in this case using peptone-free medium (PFM) to prevent $B$. thuringiensis spore germination outside the host (see Methods). Lawn leaving behavior was significantly higher for CB4856 compared to $\mathrm{N} 2$ on all tested bacterial strains and for all exposure time periods (Fig. 1; Sheet 1 in Additional files 2, 3 and 4). For both C. elegans strains, we observed a significant increase in leaving across time (Fig. 1). For both, the avoidance response towards the most pathogenic strain (B-18679) was higher than that towards the less pathogenic strain (B-18247) (Fig. 1c, d).

Our results confirm previously reported higher avoidance behavior and resistance of CB4856 compared to N2 towards one of the pathogens used in the current study, B. thuringiensis B-18247 [23]. Our findings are also consistent with two previous studies that demonstrated a higher OP50-patch leaving behavior [31] and a higher microsporidia resistance of CB4856 compared to N2 [43]. Interestingly, the opposite phenotype has been reported regarding the nematode's response to two other pathogens, $P$. aeruginosa and Serratia marcescens. In these cases, N2 rather than CB4856 produced higher resistance and behavioral avoidance towards $P$. aeruginosa $[17,19]$, and higher avoidance towards $S$. marcescens [26]. Moreover, as the more pathogenic B-18679 was more strongly avoided than the less virulent B-18247 (Fig. 1c, d), C. elegans appears to be able to differentiate between different levels of pathogenicity of the same bacterial species. In this case, the difference in pathogenicity is likely due to expression of different Cry toxins that result in different infection patterns [37]. Based on our results we expect that the N2 and CB4856-derived RIL and IL populations are likely to contain sufficiently high levels of variation for a QTL analysis of avoidance behaviors towards the four chosen bacterial strains.
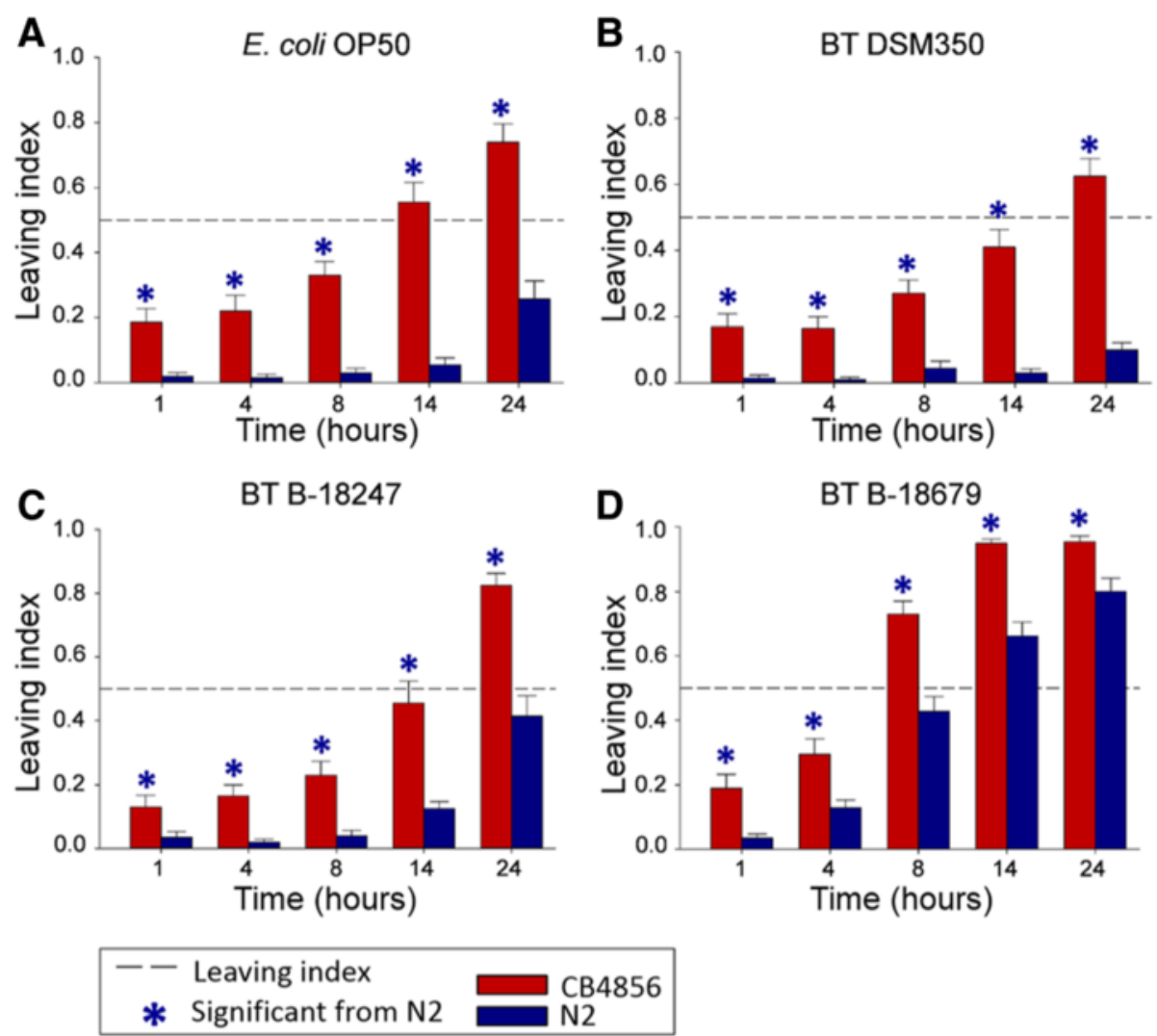

Fig. 1 Variation in lawn leaving behavior between N2 and CB4856 towards different bacterial strains. Lawn leaving behavior towards: (a) the non-pathogenic E. coli strain OP50; (b) the non-nematocidal B. thuringiensis strain DSM350; (c) the nematocidal B. thuringiensis strain B-18247; and (d) the highly nematocidal B. thuringiensis strain B-18679. The Y axis shows the leaving index for N2 (blue) or CB4856 (red), calculated as the proportion of nematodes which left the bacterial lawn; the $X$ axis indicates the different exposure time points. Asterisks denote the treatments for which leaving behavior of CB4856 is significantly different from N2. The dotted reference line shows a leaving index of 0.5. Error bars represent standard error of the mean. Raw data are given in sheet 1 of Additional file 2. Detailed statistical results are shown in Additional file 3 
Multiple QTLs and their interactions account for variation in avoidance behavior

We performed QTL analyses on C. elegans pathogen defense and revealed the genetic architecture of pathogen avoidance behavior to (i) be polygenic, (ii) include epistatically interacting loci, and (iii) incorporate general as well as pathogen-specific avoidance loci. In particular, our study simultaneously assessed the behavioral response of 200 RILs and 90 ILs [41, 42] towards four bacterial strains (two nematocidal $B$. thuringiensis and two non-nematocidal controls) at two exposure time points (14 $\mathrm{h}$ and $24 \mathrm{~h}$ ) and with three replicates per treatment combination, using the same lawn leaving assays as above for N2 and CB4856. Below we present our results of a main-effect QTL analysis of the RIL population and an analysis of interaction effects among loci for the RIL population.

The main effect QTL analysis uncovered five main regions associated with avoidance: (i) one large region in the middle of chromosome II, for which the CB4856 allele(s) increase(s) leaving behavior of the non-pathogenic bacteria only at the $24 \mathrm{~h}$ time point (Fig. 2a, b); (ii) a region on chromosome II, for which the N2 allele(s) specifically increase(s) avoidance of the more pathogenic strain B18679 at the $24 \mathrm{~h}$ time point (Fig. $2 \mathrm{~d}$ ); (iii) a region on the left arm of chromosome IV, for which the N2 allele(s) increase(s) leaving behavior towards controls and pathogens (Fig. 2a-d); (iv) a region on the right arm of chromosome
IV, for which the CB4856 allele(s) increase(s) avoidance of pathogens at both time points (Fig. $2 \mathrm{c}, \mathrm{d}$ ); and (v) a region on chromosome $\mathrm{X}$ with the strongest effect on leaving behavior towards controls and pathogens (Fig. 2a-d), mediated by the CB4856 allele(s) and including a significant time effect on the response to the pathogenic bacteria (Fig. 2c, d). This very strong $\mathrm{X}$ chromosome effect was confirmed by the ILs (Additional file 5).

Our analysis of interaction effects among QTLs, using a standard interaction model (phenotype $\sim$ time + marker $1 *$ marker2), revealed several significant intra-genomic associations with an influence on lawn leaving behavior. For $E$. coli, significant interactions were found for at least two cases (Fig. 3a): (i) between the beginning of chromosome I and the first half of chromosome $\mathrm{X}$; and (ii) between the end of chromosome II and almost the entire IV chromosome. For DSM350, we identified interaction effects between: (i) the end of chromosome IV and the first quarter of chromosome X; and (ii) the end of chromosome II and almost the entire IV chromosome (Fig. 3b). The latter seems to be specific for food patch leaving behavior as it was identified for both of the non-pathogenic bacteria (Fig. 3a, b). For the nematocidal B-18247, we found interaction effects at least between: (i) the beginnings of chromosome II and V; and (ii) the second quarter of chromosome II and the beginning of chromosome $\mathrm{X}$ (Fig. 3c). For the highly nematocidal B-18679, several interaction effects were identified including: (i) between
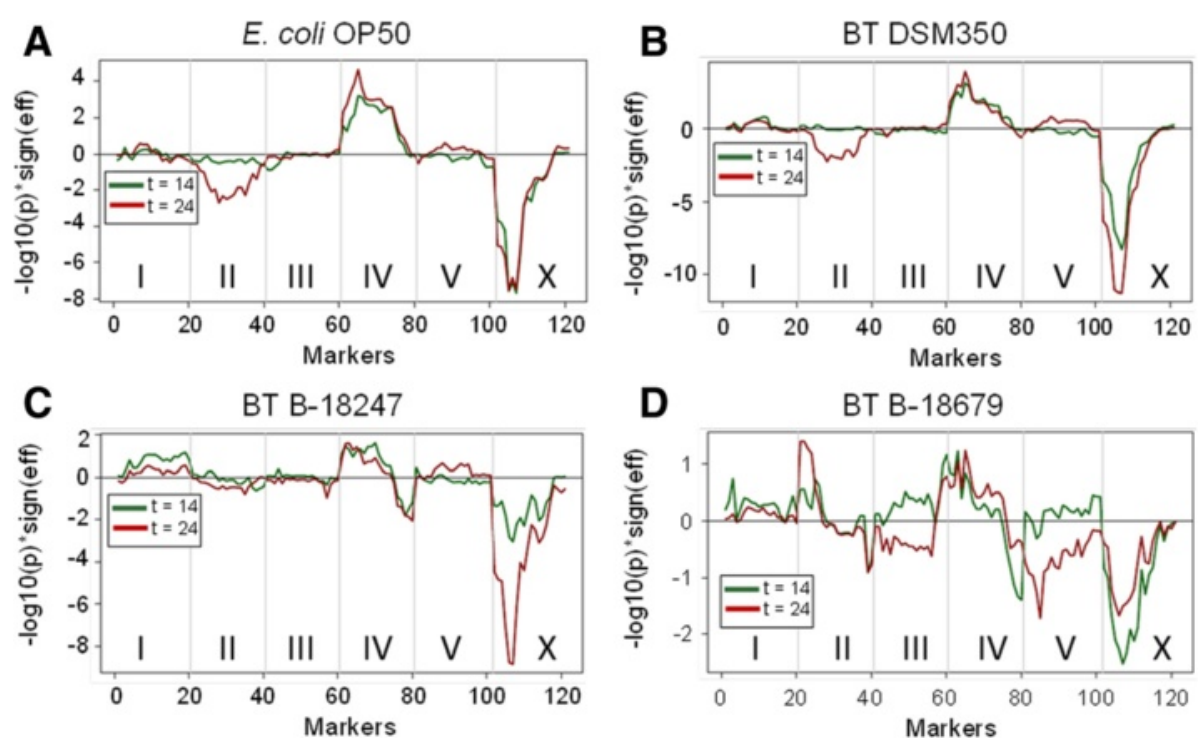

D

BT B-18679

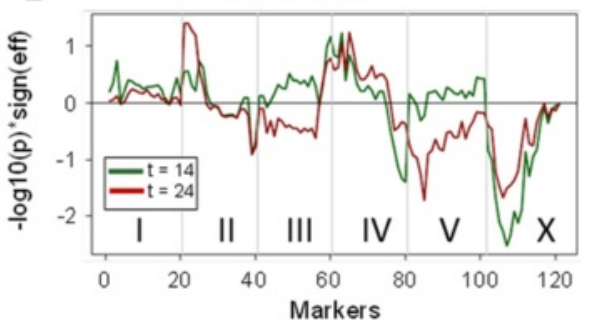

Fig. 2 QTL profiles for single marker mapping of avoidance behavior. QTL analysis of avoidance of: (a) E. coli OP50; (b) the non-nematocidal B. thuringiensis DSM350; (c) nematocidal B-18247; and (d) highly nematocidal B-18679. The X-axis shows the markers along the five autosomes and the $\mathrm{X}$ chromosome. Vertical light gray lines denote the boundaries between chromosomes. The $Y$-axis indicates the association between the chromosomal markers and variation in avoidance. Significance is indicated by - $\log 10$ of the $p$-value obtained from the linear model, which is multiplied by the sign of the effect to indicate the N2 allelic effect on avoidance. A value above 0 indicates an increase in leaving caused by the N2 allele; a value below 0 indicates an increase in leaving caused by the CB4856 allele. By convention, values above +2 or below -2 are considered to indicate a significant influence. Green and red lines show the results after either $14 \mathrm{~h}$ or $24 \mathrm{~h}$ exposure, respectively 

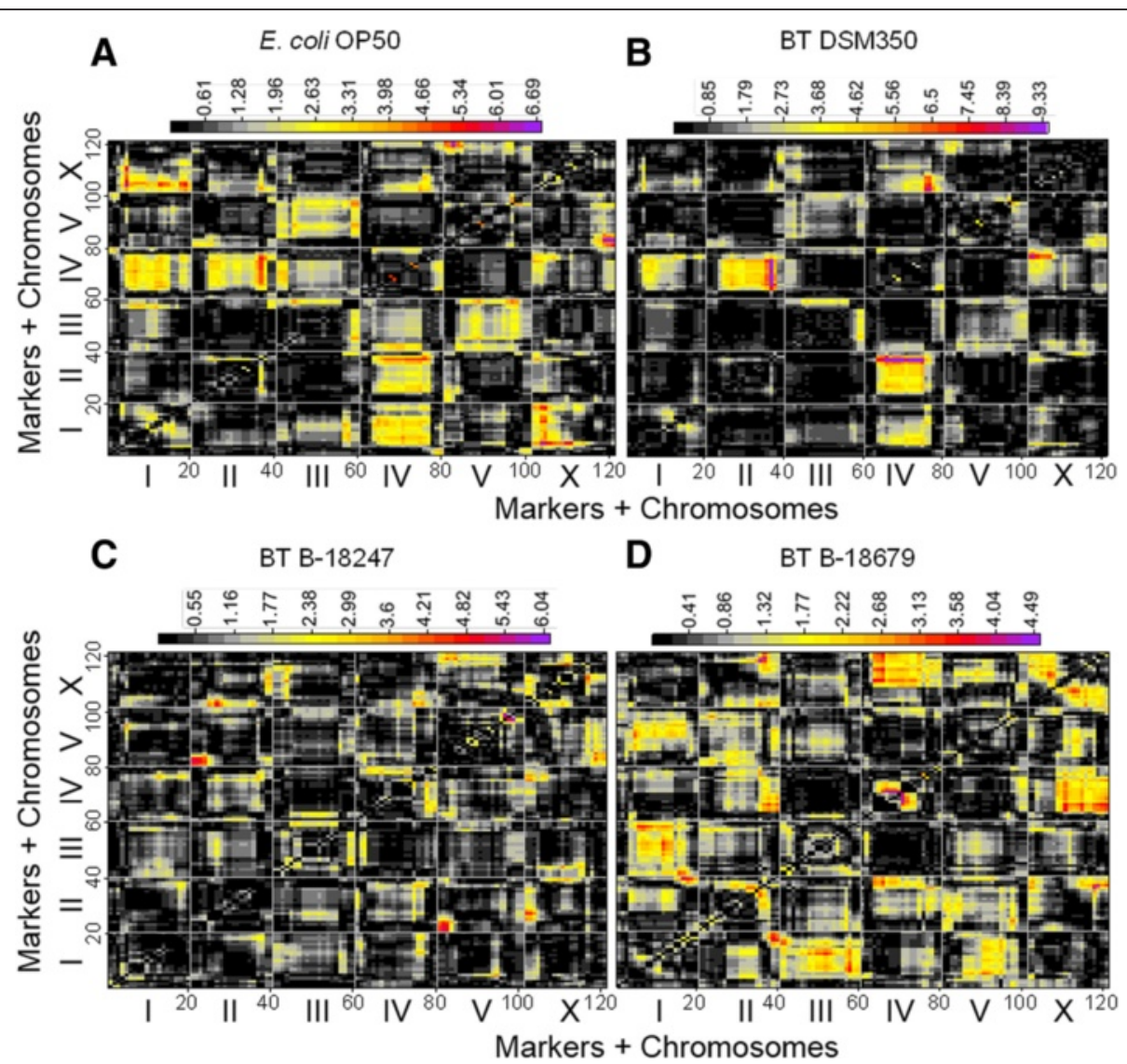

Fig. 3 Heat-map of interaction effects for avoidance behavior. Results for avoidance of: (a) E. coli OP50; (b) DSM350; (c) nematocidal B-18247; and (d) nematocidal B-18679. The distribution of markers across the genome is shown on both axes. The chromosome boundaries are indicated by the thin vertical and horizontal lines. A color legend for significance of the interaction between markers is shown on top of the panels. Significance is in - $\log 10(p)$. High significance values are given in "warm" colors with purple and red indicating the highest significances, followed by orange and then yellow. For example, in panel (c) showing the results for B-18247, the thin red area towards the bottom right indicates a significant interaction between loci from the beginning of the X chromosome (horizonal axis) and the middle of chromosome II (horizontal axis). The other slightly larger red area in the bottom right of this panel points to a significant interaction between the beginning of chromosome $V$ (horizontal axis) and the beginning of chromosome II (vertical axis)

the ends of chromosome I and II; and (ii) between the second quarter and the middle of chromosome IV (Fig. 3d). Interestingly, the $\mathrm{X}$ chromosome region with the strongest influence in the main effect model (Fig. 2) only contributed to very few significant interaction effects. One of these is an interaction with a chromosome I region,mediating avoidance of $E$. coli OP50 (Fig. 3a), and another one with a chromosome IV region, influencing avoidance of DSM350 (Fig. 3b).

Taken together, our results demonstrate that pathogen avoidance has a complex genetic architecture in C. elegans, which overlaps with, but differs from the response to non-pathogenic microbes. In particular, pathogen defense traits are related to the response to non-pathogenic bacteria, because they are affected by the same loci. Defense is thus in part determined by the general response to microbes, whereby pathogenicity of the bacteria may simply elevate the response mediated by a particular locus, as indicated for the X chromosome QTL (Fig. 2). Moreover, our results for the pathogen-specific QTLs are consistent with the previous finding that pathogen defense in invertebrate animals seems to rely on few loci and involve epistatic interactions among them [44, 45], possibly as a consequence of reciprocal coevolution among host and pathogens [44]. It will be a rewarding challenge for the future to characterize the genes underlying the pathogenspecific QTLs. Interestingly, the main effect QTL on the $\mathrm{X}$ chromosome was previously implicated in lawn leaving behavior with similar allelic effects towards the nonpathogenic E. coli OP50 (i.e., the CB4856 allele increases avoidance; [31]) but with opposite allelic effects towards $P$. aeruginosa (i.e., the N2 allele increases avoidance; [17-19]). In these cases, the QTL effect on chromosome $\mathrm{X}$ could be associated with variation in the gene $n p r-1[17-19,31]$ and, at least towards E. coli, additionally the catecholamine receptor gene tyra-3 [31]. 
The $n p r-1$ gene affects defense against $B$. thuringiensis The gene $n p r-1$ was previously linked to the strong-effect QTL on chromosome X [17], which we found in the current study to influence $C$. elegans defense towards $B$. thuringiensis in the opposite way than that towards $P$. aeruginosa. We therefore specifically tested whether this gene is indeed responsible for the contrasting phenotypes in pathogen avoidance and resistance.

We first studied the role of $n p r-1$ in lawn leaving behavior on E. coli and B. thuringiensis using two mutants (npr1(ad609), npr-1(ur89)), which were both previously shown to decrease pathogen avoidance behavior against $P$. aeruginosa $[17,18]$. Another gene from the left arm of chromosome $\mathrm{X}$ was previously demonstrated to influence food patch leaving behavior, namely tyra-3, which encodes a tyramine receptor homologue [31]. Therefore, we further tested its involvement in pathogen defense with the knock-out mutant tyra-3(ok325).

Analysis of the two mutant npr-1 alleles (npr-1(ad609), $n p r-1(u r 89))$ yielded different results in avoidance and resistance (Figs. 4 and 5; sheets 2-4 of Additional files 2, 6, 7 and 8). In particular, avoidance behavior of npr-1(ur89) was similar to CB4856 but higher than that of N2 (Fig. 4, Additional file 6). In contrast, npr-1(ad609) always produced a low leaving rate, similar to N2 and clearly different from CB4856. On the highly pathogenic B-18679, avoidance behavior was extremely high at both time points without any significant differences among the $C$. elegans strains (Fig. 4d). In addition, the tyra-3 mutant consistently showed a similar behavioral response to $\mathrm{N} 2$, irrespective of the bacterium and the exposure time (Fig. 4, Additional file 6).

The $n p r-1$ alleles produced similarly contrasting effects on survival rate, which is often used as a proxy for nematode immunity. For the RIL/IL parental strains, we found that CB4856 showed significantly higher resistance than N2 on both nematocidal B. thuringiensis strains (Fig. 5; Additional files 7, 8 and 9). Moreover, the npr-1(ur89) mutant was significantly more resistant than N2 and as resistant as CB4856 on both nematocidal pathogens, whereas
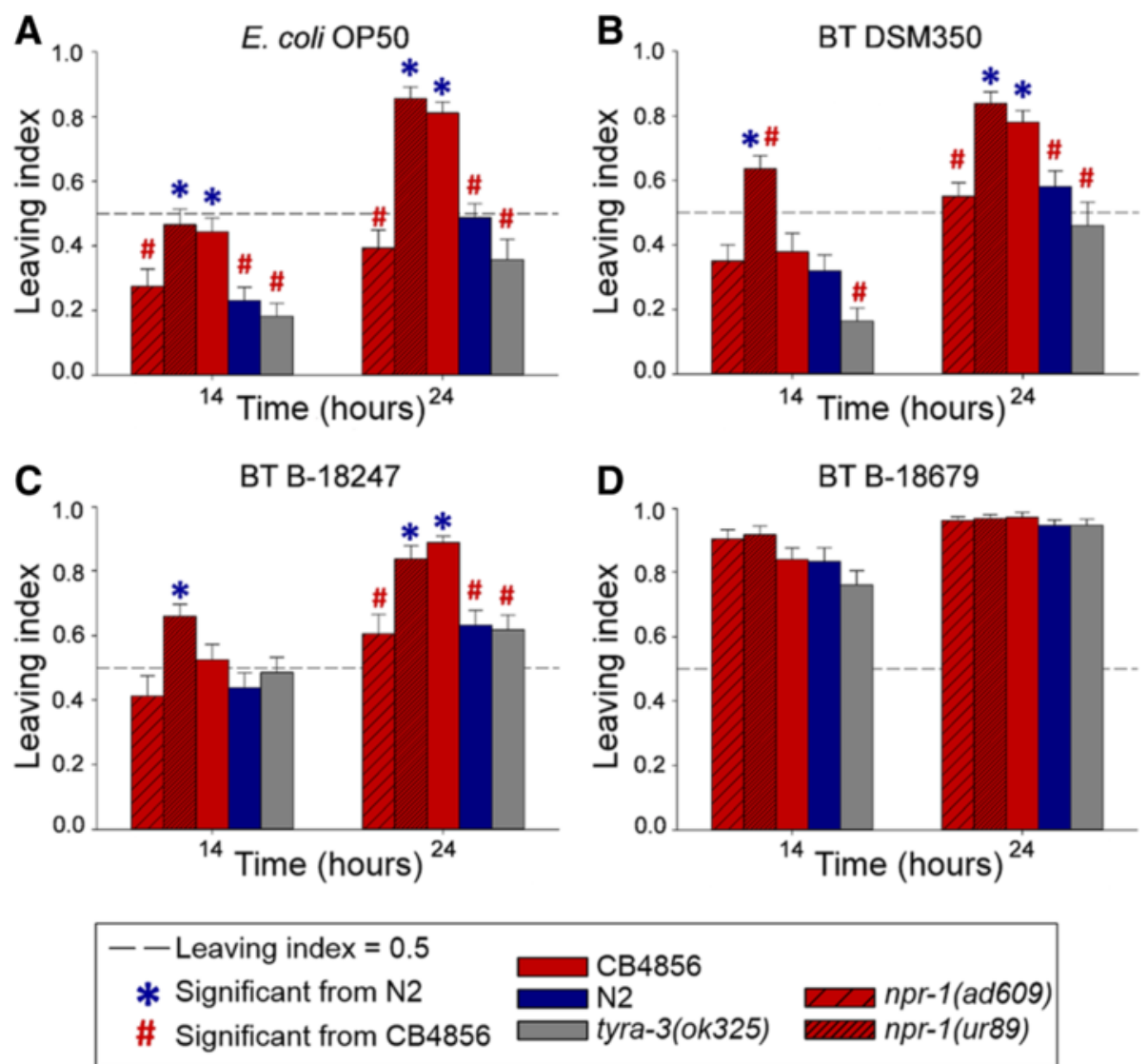

Fig. 4 Lawn leaving behavior for N2, CB4856, and different mutants towards E. coli and B. thuringiensis. Results for avoidance of: (a) E. coli OP50; (b) non-nematocidal B. thuringiensis DSM350; (c) nematocidal B. thuringiensis B-18247; and (d) highly nematocidal B-18679. The Y axis shows the proportion of escaped worms (i.e., leaving index); the $X$ axis shows the two time points at which leaving was scored. The asterisk $\left({ }^{*}\right)$ is shown for the treatments which differ significantly from N2 whereas the hash sign (\#) indicates significant differences from CB4856. The dotted reference line shows a leaving proportion of 0.5 for orientation among sub-panels. Error bars represent standard error of the mean. Raw data are given in sheet 2 of Additional file 2; detailed statistical results in Additional file 6 


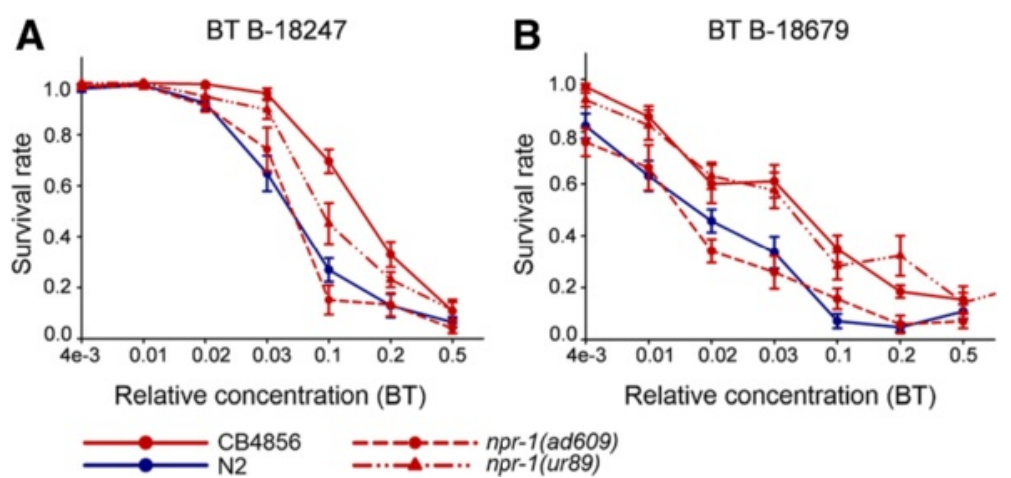

Fig. 5 Survival of N2, CB4856, npr-1 (ad609) and npr-1(ur89) on nematocidal B. thuringiensis. Results for: (a) nematocidal B-18247; and (b) highly nematocidal B-18679. Survival on the $Y$ axis was plotted against relative $B$. thuringiensis concentration on the $X$ axis. Error bars represent standard error of the mean. The raw data are provided in sheet 4 of Additional file 2; the statistical results in Additional file 8

$n p r-1$ (ad609) was as susceptible as $\mathrm{N} 2$ on both pathogenic strains (Fig. 5, Additional file 8). None of the C. elegans strains showed any mortality under control conditions (results not shown).

We conclude that the two mutant npr-1 alleles produce opposite effects on both behavioral avoidance of the four bacterial strains and also resistance against nematocidal $B$. thuringiensis. Consequently, variation in $n p r-1$ may only partially explain the strong main effect QTL on the X chromosome. The difference between the two $n p r-1$ alleles in avoidance and resistance of $B$. thuringiensis is surprising, because both alleles behaved similarly in previous studies investigating resistance against $P$. aeruginosa $[17,18]$. Yet the two alleles carry different mutations: $n p r-1($ ad609) two in exons 2 and 3, whereas the mutation of $n p r-1$ (ur89) falls into exon 3 (http://www.wormbase.org). The exact reasons for the different effects of these alleles clearly deserve further investigation in the future, ideally including additional loss-of-function and also reduced-function $n p r-1$ alleles in combination with a tissue-specific analysis of the mutational effects. We further conclude that the tyra-3 gene does not appear to influence the assayed phenotypes (Figs. 4 and 5), including avoidance of E. coli OP50, which was however previously demonstrated in a separate study [31]. The difference in results could be due to variation in experimental approaches. For example, we directly characterized leaving behavior, whereas the previous study scored activity as a proxy for leaving behavior [31].

\section{Contrasting effect of $n p r-1$ on defense against Pseudomonas aeruginosa}

We sought to confirm the previously published finding [17-19] that the wild-type N2 produces higher resistance and stronger avoidance behavior towards the pathogen $P$. aeruginosa PA14 than the Hawaiian strain CB4856 and two npr-1 mutants [17-19], thus contrasting with our above results for B. thuringiensis. Here, we specifically re-evaluated these previous results under our laboratory conditions and assay protocols, using the peptone-rich NGM plates required for expression of $P$. aeruginosa virulence. We first used the lawn leaving assay to assess the avoidance response against PA14 at different exposure time points. Consistent with previous findings, the $n p r-1$ mutants and CB4856 showed significantly lower PA14 pathogen avoidance than N2 across all time points (Fig. 6; sheets 5 and 6 of Additional files 2, 10, 11 and 12). For the $48 \mathrm{~h}$ time point the mutant npr-1(ad609) even had a lower leaving response than CB4856 (Fig. 6b). On OP50, leaving behavior was similar for all C. elegans strains at all time points except at time point $14 \mathrm{~h}$, when the mutant $n p r-1$ (ad609) showed a more pronounced leaving behavior than N2 (Fig. 6a, Additional file 12). In this assay, we also included a tyra-3 mutant, which expressed a similar leaving response to $\mathrm{N} 2$ under all conditions except at time point $48 \mathrm{~h}$, where its leaving response against PA14 was reduced in comparison to N2, but still significantly higher than that of the remaining strains (Fig. 6b, Additional file 12).

We next evaluated the effect of $n p r-1$ on resistance against PA14 using standard C. elegans survival assays. All strains survived less on the pathogen PA14 than on the control OP50 (Fig. 7; sheet 7 of Additional files 2 and 13). On the pathogen, N2 was significantly more resistant than all other tested strains (Fig. 7b, Additional file 14).

We conclude that $n p r-1$ directly influences avoidance of PA14, in agreement with previous work [17-19]. In these studies, $n p r-1$ was suggested to affect PA14 resistance either as a consequence of hyperoxia avoidance behavior only (proposed by Reddy et al., [17]) or through both hyperoxia avoidance and the regulation of physiological immune responses (proposed by Styer et al., [18]).

Our results further demonstrate that the two C. elegans wild-type strains express opposite phenotypes on the two tested pathogens and that this contrast may be mediated at least partially by $n p r-1$, as one of the $n p r-1$ alleles also produces an opposite phenotype relative to N2. Such opposite effects in the wild-type strains indicate specific 


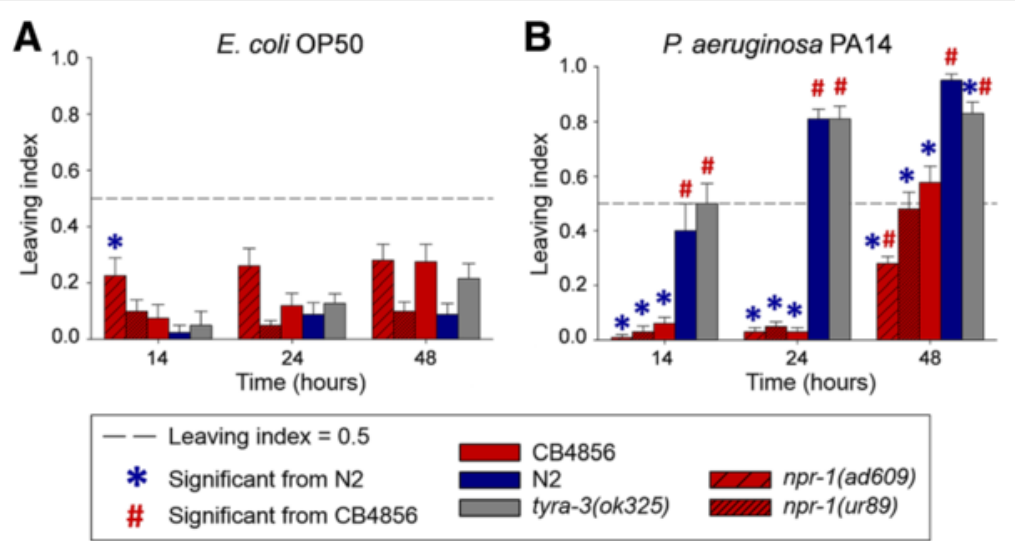

Fig. 6 Lawn leaving behavior for N2, CB4856 and different mutants towards E. coli and P. aeruginosa. Results for: (a) E. coli OP50; and (b) P. aeruginosa strain PA14. The $Y$ axis shows the proportion of escaped worms; the $X$ axis shows the three time points at which leaving was scored ( $12 \mathrm{~h}, 24 \mathrm{~h}$, and $48 \mathrm{~h}$ ). The asterisk (*) denotes the treatments which differ significantly from N2, whereas the hash sign (\#) indicates significant differences from CB4856. The dotted reference line shows a leaving proportion of 0.5 . Error bars represent standard error of the mean. The raw data are provided in sheet 6 of Additional file 2; detailed statistical results in Additional file 12

interactions with pathogens. The underlying genetics for such specificities have not yet been explored for behavioral immune defense. Some information is available for physiological and cellular immune specificities. In the higher vertebrates, such specificities can be mediated by components of the adaptive immune system such as the highly variable receptors of the major histocompatibility complex or the highly variable $\mathrm{T}$ and $\mathrm{B}$ cell receptors. Similar specificities have also been recorded in invertebrates $[1,46]$, where they may be due to different immune signaling cascades. For example, in Drosophila, the immune deficiency pathway appears to be more important in the systemic response to Gram-negative bacteria, whereas the Toll pathway is more important towards Gram-positive bacteria and fungi [47]. Moreover, in C. elegans, mutations in the egl-9 and tol-1 gene enhance resistance against some pathogens, while simultaneously increasing susceptibility to other pathogens (see introduction and $[5-8,10]$ ). Our study thus provides one of the few examples which demonstrate that a single gene, in this case the neuropetide $\mathrm{Y}$ receptor homolog gene $n p r-1$, produces contrasting pathogen specificities in an invertebrate.

At the same time, it is less clear how exactly npr-1 causes these contrasting phenotypes. Previous work on nematode social behavior demonstrated that $n p r-1$ influences worm aggregation, lawn bordering and clumping through its effect on aerotaxis behavior. The two tested $n p r-1$ alleles and also that of the CB4856 strain result in a preference towards lower oxygen concentrations usually found at the edge of the bacterial lawn [48, 49], whereas the $\mathrm{N} 2$ allele shows no such preference. A similar difference in aerotaxis behavior may also explain the reduced $P$. aeruginosa avoidance and resulting higher susceptibilities of the CB4856 and npr-1 mutant strains, which remain in longer contact with the harmful pathogen, because the bacterial lawn boundaries show the preferred lower oxygen concentrations [17]. An involvement of such aerotaxis behavior in the $B$. thuringiensis
A

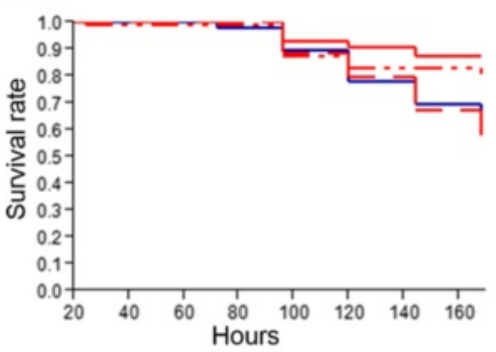

B

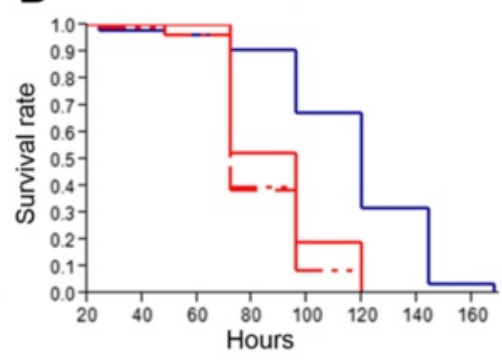

CB4856 - - - - npr-1(ad609)

Fig. 7 Survival of N2, CB4856, npr-1(ad609) and npr-1(ur89) on E. coli and P. aeruginosa. Results for: (a) E. coli OP50; and (b) P. aeruginosa strain PA14. Survival ( $Y$ axis) was plotted against time in hours ( $X$ axis). We used the Kaplan-Meier method to calculate survival fractions. The raw data are given in sheet 7 of Additional file 2; statistical results in Additional files 13 and 14 
response of CB4856 and one of the $n p r-1$ mutants would then require that the preferred oxygen concentration is outside of the bacterial lawn, which is unlikely to be the case (assuming higher oxygen concentrations outside, where no oxygen is consumed by proliferating bacteria). Thus, it is conceivable that CB4856 and the one npr-1 mutant are directly responding to a compound produced by $B$. thuringiensis, and that this response is less pronounced in the N2 strain and the other npr-1 mutant. In this context, it is worth noting that the high variation between N2 and CB4856 in their leaving response towards the control E. coli OP50 was only observed on peptone free PFM but not the peptone-rich NGM assay plates (Figs. 1a and 4a versus Figs. 6a and Additional file 10). This is most likely explained by bacterial proliferation, which is possible on NGM but not PFM assay plates. In turn, the lack of proliferation on the PFM plates is unlikely to coincide with large variation in oxygen concentration, such that an aerotaxis response should be less pronounced under these conditions. Yet, a non-proliferating, static bacterial population may produce particular metabolites, which then could have induced the CB4856 avoidance response.

\section{npr-1 influences the transcriptomic response to $B$. thuringiensis and $P$. aeruginosa}

To explore the mechanisms underlying the npr-1 mediated contrasting effects on immune defense, we assessed whether $n p r-1$ differentially affects gene expression in the presence of either of the two pathogens. Using RNAseq we compared the transcriptomes of the $\mathrm{N} 2$ and npr-1(ur89) strains exposed to either the nematocidal $B$. thuringiensis B-18247, the pathogenic $P$. aeruginosa PA14, or the control E. coli OP50. We chose the mutant npr-1(ur89) because it showed differential leaving behavior and survival on both pathogens compared to N2 (Figs. 4 and 6). Exposure experiments were performed on Agar plates fully covered with bacterial lawns, thus reducing possible avoidance behaviors and producing comparable levels of lawn occupancy for the worms from the various treatment combinations. RNA transcript levels were characterized at two time points, $12 \mathrm{~h}$ and $24 \mathrm{~h}$ of pathogen exposure. We used principal component analysis (PCA) to explore which experimental factors generated different transcriptional responses. The first principal component indicated that the two nematode strains vary in their transcriptional signature to all three bacteria (Fig. 8). The second principal component highlights variation across several additional factors. The strongest effect stems from exposure time (light versus dark colors; Fig. 8). Additional influences can be seen for pathogen exposure versus the corresponding control, especially at the later time point (filled versus open symbols of the same type; Fig. 8) and also a clearly distinct signal after 24 $\mathrm{h}$ exposure to PA14 compared to all other conditions

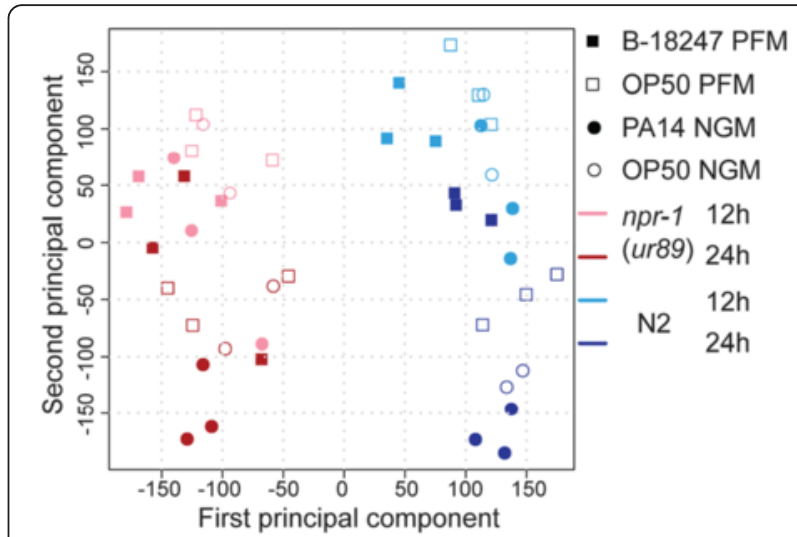

Fig. 8 Principal component analysis of transcriptomic variation. Variation is assessed for npr-1 (ur89) and N2 upon exposure to pathogens (B-18247 and PA14) and the control bacterium OP50. N2 is shown in blue whereas npr-1(ur89) is shown in red. Light and dark colors indicate the early and late time point, respectively (12 h versus $24 \mathrm{~h}$ ). Filled and open symbols denote exposure to pathogens (B-18247 and PA14) and control bacteria (OP50), respectively. NGM is the nematode growth medium enriched with peptone for the PA14 assay plates, PFM refers to peptone-free NGM used for the B-18247 assay plates

(filled dark colored circles towards the bottom of the graph; Fig. 8). These latter differences are more pronounced for $\mathrm{N} 2$ than the npr-1 mutant, especially as $\mathrm{N} 2$ produces clearly distinct treatment signatures at the later $24 \mathrm{~h}$ time point (i.e., clearly separated dark blue open and filled circles and squares; Fig. 8). One possible reason for lower differentiation in the $n p r-1$ mutant may be a lower number of differentially expressed genes compared to the N2 strain. This was indeed the case, especially upon pathogen exposure (Table 1), suggesting that mutations in $n p r-1$ somehow compromise the signalling response to pathogen infection.

To identify groups of co-regulated genes, we next performed K-means clustering on the significant gene sets. The resulting eight clusters confirm that the transcriptional response is influenced by the $C$. elegans strain, the pathogen strain and also the exposure time point (Fig. 9; Additional file 15). In detail, clusters 1, 2, 3, and 4 refer to genes with strong differential expression upon exposure to only pathogenic $B$. thuringiensis B-18247 in only the C. elegans N2 strain, but neither the npr-1(ur89) mutant on the same pathogen nor any of the other treatments with $P$. aeruginosa (e.g., the stronger the color intensity in Fig. 9, the stronger the expression difference between pathogen versus non-pathogen exposure). This result again highlights that the $n p r-1$ mutant shows generally lower responsiveness in inducible gene expression (i.e., most clusters do not show high color intensity in Fig. 9). Clusters 1, 2, 3 and 4 only responded to the pathogen B-18247, and clusters 7 and 8 only or at least predominantly to PA14. Two clusters are specific to expression variation at the $12 \mathrm{~h}$ time point, in both cases upon 
Table 1 Number of up- and down-regulated genes in the N2 and npr-1(ur89) strains

\begin{tabular}{|c|c|c|c|c|}
\hline \multirow[b]{2}{*}{ C. elegans } & \multicolumn{2}{|c|}{ B-18247 } & \multicolumn{2}{|l|}{ PA14 } \\
\hline & $12 \mathrm{~h}$ & $24 \mathrm{~h}$ & $12 \mathrm{~h}$ & $24 \mathrm{~h}$ \\
\hline \multicolumn{5}{|l|}{ Up-regulated } \\
\hline N2 & 1393 & 1094 & 222 & 529 \\
\hline npr-1(ur89) & 179 & 191 & 188 & 352 \\
\hline \multicolumn{5}{|c|}{ Down-regulated } \\
\hline $\mathrm{N} 2$ & 2384 & 458 & 81 & 1233 \\
\hline npr-1(ur89) & 117 & 70 & 58 & 370 \\
\hline
\end{tabular}

RNAseq was used to assess variation in gene expression among the $C$. elegans $\mathrm{N} 2$ and npr-1(ur89) mutant strain in response to exposure to nematocidal $B$. thuringiensis $\mathrm{B}-18247$ and $P$. aeruginosa $\mathrm{PA} 14$, always relative to the respective E. coli OP50 control. Gene expression variation was studied at two time points, $12 \mathrm{~h}$ and $24 \mathrm{~h}$ after initial exposure. The results are shown separately for the up- and down-regulated genes (top and bottom part of the table, respectively)

exposure to B-18247 (i.e., clusters 1 and 3), whereas four clusters indicate a more pronounced response at the later 24 h time point, either towards only B-18247 (clusters 2 and 4) or only PA14 (clusters 7 and 8). The remaining two clusters highlight patterns of early or continuous transcriptional response towards B-18247 and late transcriptional response towards PA14 (clusters 5 and 6; Fig. 9). None of the identified clusters showed an opposite gene expression pattern between either $\mathrm{N} 2$ and the $n p r-1 \mathrm{mu}-$ tant (e.g. up in N2 and down in the $n p r-1$ mutant) or the two pathogens (e.g. up after B. thuringiensis but down after $P$. aeruginosa exposure). Taken together, clusters 5 and 6 appear to encompass a general defense response against both pathogens, whereas the clusters $1,2,3$, and 4 define the specific response to B-18247 and clusters 7 and 8 that to PA14. Therefore, the latter two groupings are likely to account for the observed npr-1 dependent defense differences towards the two pathogens. We thus conclude that the considered mutation in the $n p r-1$ gene causes a decreased transcriptomic response to the two pathogens, which induce overlapping and distinct sets of differentially expressed genes.

\section{Different functions and signaling processes are affected} by the pathogen-dependent $n$ pr-1-specific transcriptome We used enrichment analysis as a statistical tool to explore the possible functions of the differentially regulated gene clusters. Four types of enrichment analyses were performed, which aim to identify significant over-representation of (i) genes with a specific gene ontology (GO) term (GO term analysis); (ii) customized nematode-specific gene sets, inferred from previous gene expression analyses and based on the program EASE (EASE analysis); (iii) genes with specific transcription factor-binding motifs (Motif analysis), and (iv) expression QTLs (eQTLs). The customized enrichment analysis with the program EASE [50] was based on a large database of all previous $C$. elegans transcriptome

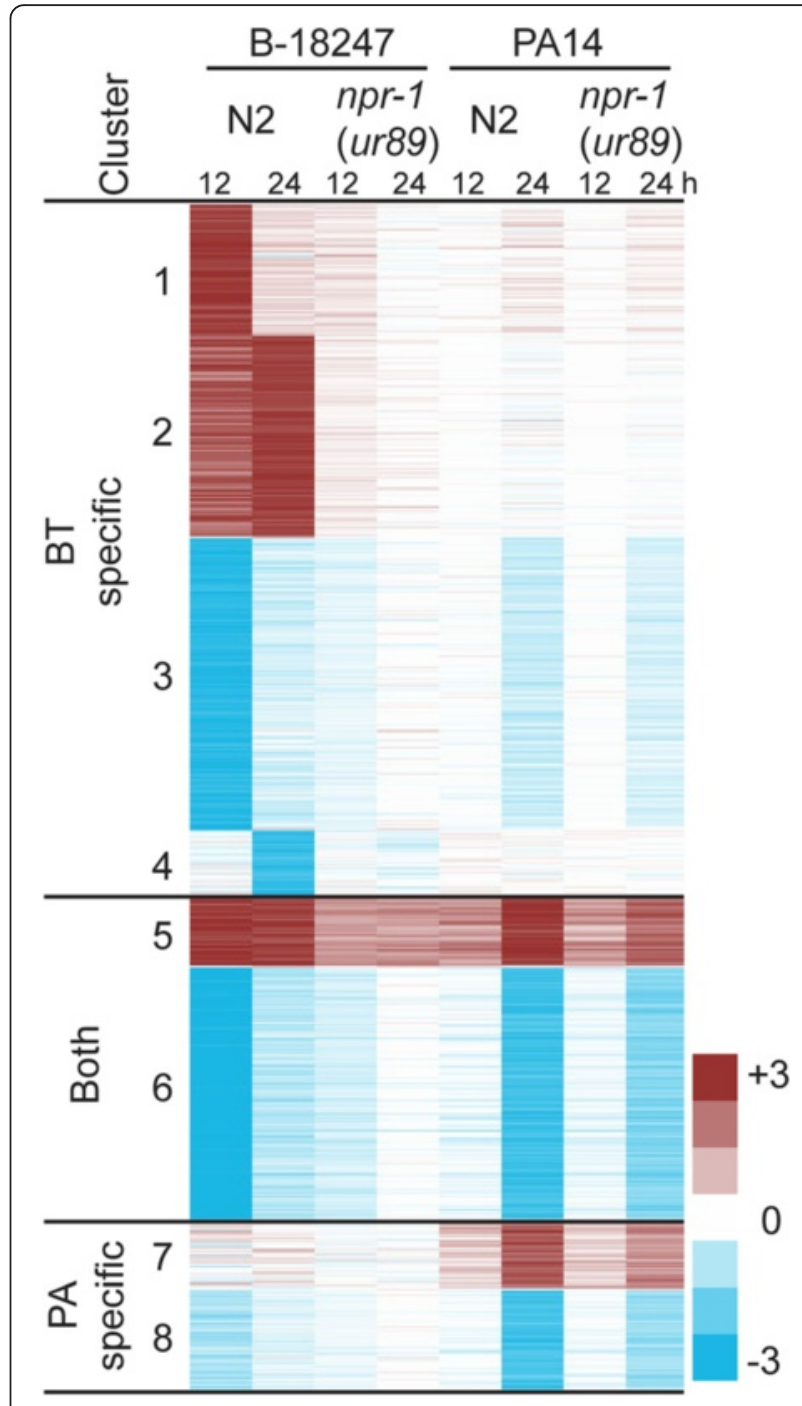

Fig. 9 Co-regulation of the differentially expressed genes. K-means cluster analysis yielded eight clusters of co-regulated genes, as indicated by the numbers on the left. The top of the graph shows the different treatment conditions, including C. elegans strain, pathogen strain and exposure time point. Red refers to up-regulated genes, whereas blue to down-regulated genes, always upon pathogen exposure relative to the corresponding E. coli OP50 control. High color intensities indicate strong expression differences to the control (see legend in the bottom right corner). The complete results are given in Additional file 15

studies, WormExp [51], which we collated from published work. These studies investigated differential gene expression (i) across development, (ii) in specific tissues, (iii) in worms with defined mutations or subjected to RNAiknockdown of specific genes, or (iv) upon exposure to environmental stimuli such as pathogens, heavy metals, and other chemical compounds [51]. The GO term and Motif analyses were based on published methods, such as DAVID $[52,53]$ and AMD [54]. Analysis of eQTL enrichment expression differences, using the eQTL database collated from different previous eQTL analyses, all based on RIL panels 
derived from N2 and CB4856 as parental lines [41, 55-60], thus potentially allowing us to link the identified QTLs to the expression variation inferred against pathogens. The results are summarized in Figs. 10 and 11, Table 2, Additional files 16 and 17, and explained in more detail below.

We first focus on the general defense response against the two pathogens, which is defined by two clusters (i.e, clusters 5 and 6; Fig. 9). These clusters are enriched for genes previously implicated in C. elegans pathogen defense. These include genes involved in carbohydrate binding
(Fig. 10a), most likely mediated by $\mathrm{C}$ type lectin-like genes, many of which underlie this GO term (Additional file 17 under GO) and which are up-regulated across treatment conditions (Fig. 11a) and have repeatedly been implicated in C. elegans immunity, possibly as pathogen recognition receptors or antimicrobials [11, 61-64]. These two clusters are also enriched for genes which were previously shown to respond to exposure to the same pathogens and other types of stressors, such as heavy metals, osmotic stress, or pesticides (Fig. 10b). The upregulated genes appear to be

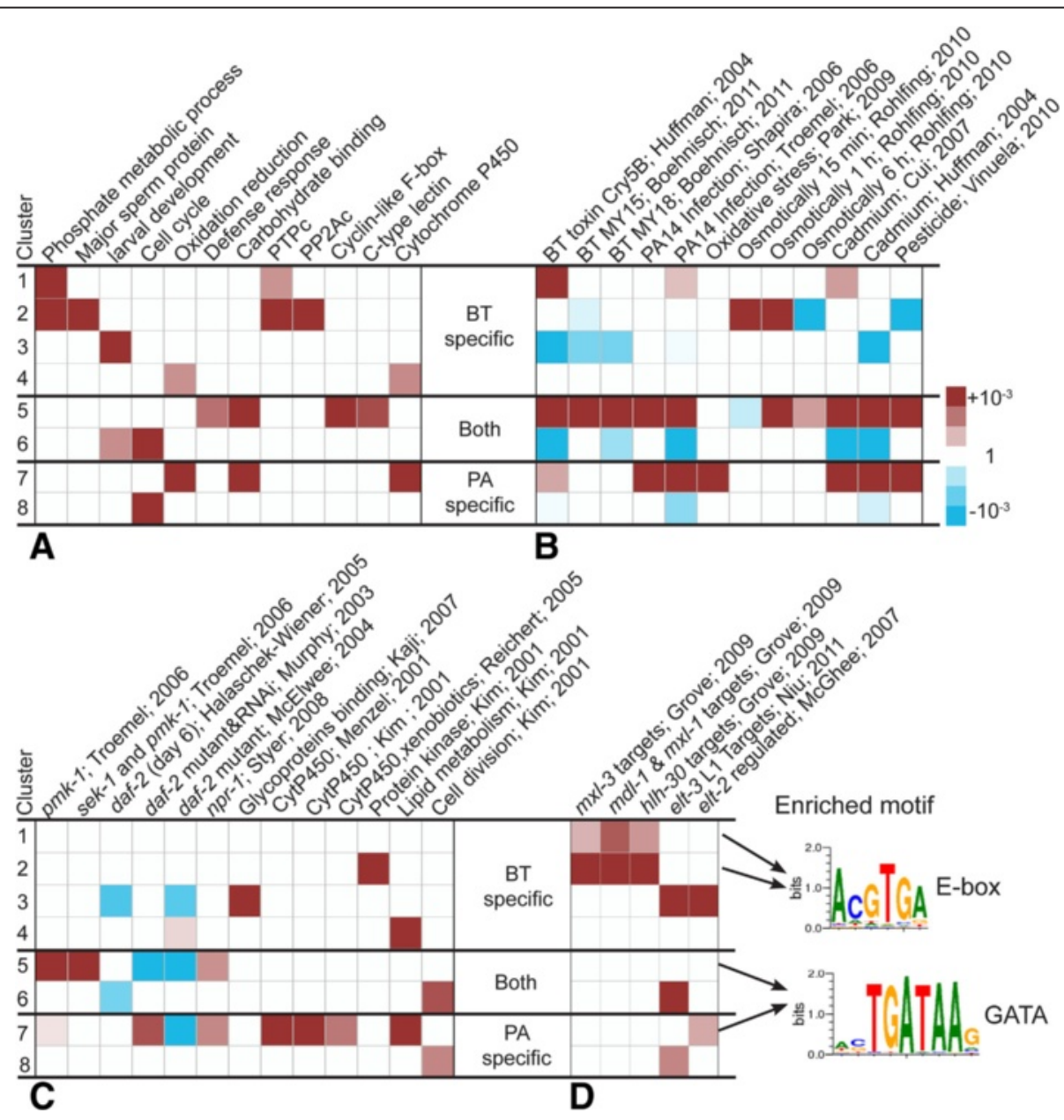

Fig. 10 Functional consequences of gene expression variation between npr-1(ur89) and N2 upon pathogen exposure. a Enrichment of gene ontology (GO) terms. The shown terms were significant with FDR $<0.05$ (Additional file 17). b Overview of enrichment of pathogen- and stressinduced gene sets, inferred from EASE analysis on the various clusters of co-regulated genes. The significantly enriched gene sets are indicated on the top and include - from left to right - differentially expressed genes upon exposure to (i) B. thuringiensis Cry5B toxin [89]; (ii)-(iii) the same B-18247 strain used in the current study in C. elegans isolate MY15 or MY18 [90]; (iv)-(v) the same PA14 strain used here [66, 91]; (vi) Oxidative stress response [92]; (vii)-(ix) Osmotic induction [93]; (x)-(xi) Heavy metal Cadmium dysregulated genes [89, 94]; (xii) Pesticide influence [58]. c Overview of enriched gene sets for selected immunity pathways and general categories, including (i)-(ii) the p38 MAPK pathway (pmk-1 and sek-1 targets; [91]); (iii)-(v) insulin signalling (daf-2 targets; [95-97]); (vi) npr-1 targets [18]; (vii) Glycoproteins; (viii)-(x) Cytochrome P450 [98-100]; (xi) Protein kinase [98]; (xii) Lipid metabolism [98]; (xiii) Cell division [98]. d Enrichment of Ebox and GATA motifs and their transcriptional targets. The enriched gene sets were inferred with EASE and are indicated at the top, including differentially expressed genes in mutants of (i)-(iii) E-box transcriptions factors [101] or (iv)-(v) GATA transcription factors ELT-2 and ELT-3 [102, 103]. Enriched transcription factor binding motifs were inferred with AMD and are shown on the right (Additional file 17). In all panels, the clusters are given on the very left and are identical to those in Fig. 9. Red color indicates an enrichment for upregulated genes per gene set, blue that for down-regulated genes per gene set. Color intensity corresponds to the significance level, inferred by EASE analysis (see scale at the right side) 


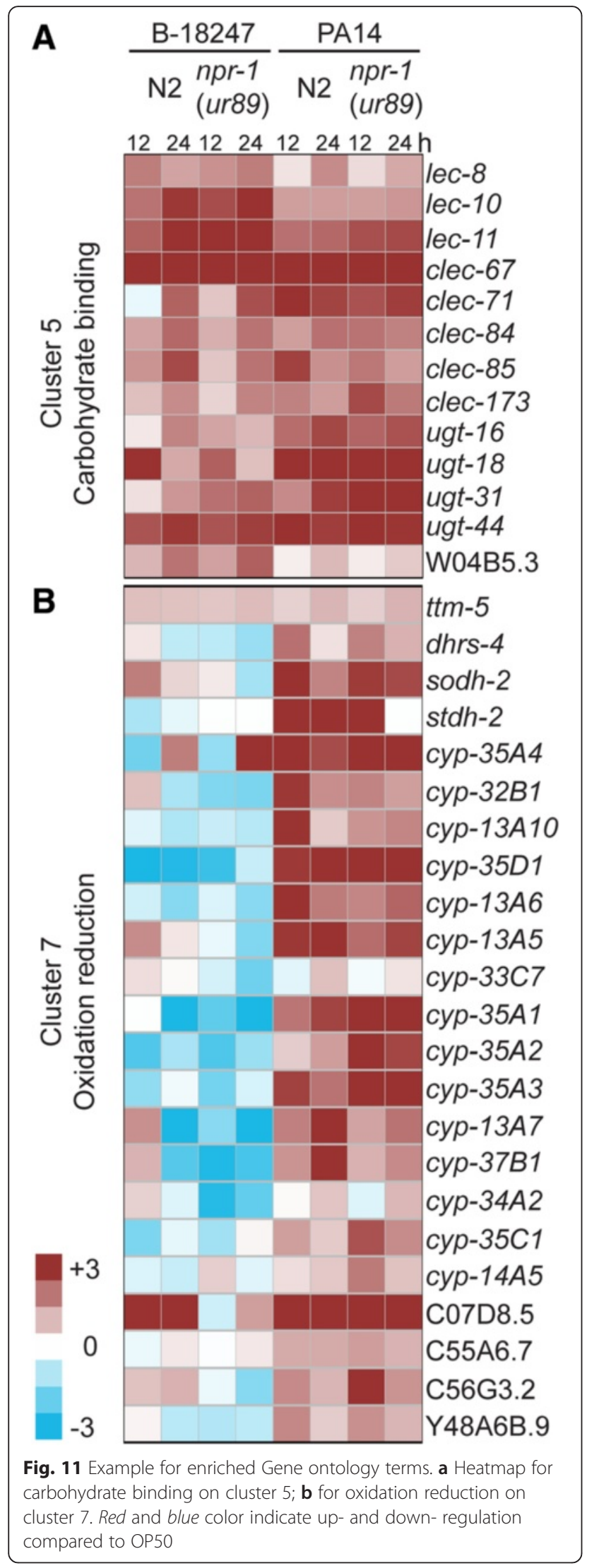

Table 2 eQTL enrichment analysis on identified expression clusters

\begin{tabular}{|c|c|c|c|}
\hline \multirow{2}{*}{$\begin{array}{l}\text { Expression } \\
\text { cluster }\end{array}$} & \multicolumn{2}{|c|}{ Location of eQTL } & \multirow[t]{2}{*}{ eQTL set } \\
\hline & Chromosome & Approximate position & \\
\hline 1 & V & $3.7 \mathrm{M}$ & Rockman \\
\hline 2 & IV & $6.6 \mathrm{M}$ & Viñuela (old) \\
\hline 3 & $x$ & $15.5 \mathrm{M}$ & Rockman \\
\hline 4 & - & - & - \\
\hline \multirow[t]{5}{*}{5} & $x$ & $2.3 \mathrm{M}$ & Rockman \\
\hline & $x$ & $5.8 \mathrm{M}$ & Rockman \\
\hline & $x$ & $10.9 \mathrm{M}$ & Rockman \\
\hline & IV & $6.3 \mathrm{M}$ & Rockman \\
\hline & III & $1.9 \mathrm{M}$ & Rockman \\
\hline 6 & I & $5.0 \mathrm{M}$ & Viñuela (juvenile) \\
\hline \multirow[t]{2}{*}{7} & $x$ & $15.7 \mathrm{M}$ & Rockman \\
\hline & V & $12.3 \mathrm{M}$ & Rockman \\
\hline 8 & | & $3.9 \mathrm{M}$ & Rockman \\
\hline
\end{tabular}

eQTL enrichment analysis was performed to identify significant overlaps between the genes underlying a specific cluster in our analysis (first column; see also Fig. 9) and previously characterized gene sets that define particular eQTLs (last column). Such overlaps can then be linked to the specific QTL regions within the genome (second and third column), which may then contain regulatory elements important for the expression variation in our study and which may also have been identified as QTLs for the observed variation in behavioral immune defense (Figs. 2 and 3). For further details see Additional file 16. No significant enrichment was found for cluster 4

controlled by two of the main C. elegans immunity signalling cascades, the p38 MAPK and the insulin-like receptor pathways (Fig. 10c) [2], and also the $n p r-1$ gene (Fig. 10c) [18]. They also show an enrichment in their promotor sequences for a GATA binding motif, although not for known targets of the GATA transcription factors ELT-2 and ELT-3 (Fig. 10d). They are also enriched for gene sets defined by eQTLs on chromosome I, III, the middle of chromosome $\mathrm{IV}$, and the left arm and the middle of chromosome X (Table 2). One of the enriched X chromosome eQTLs encompasses the npr-1 gene, another the gene sek-1 of the p38 MAPK cascade, and the one on chromosome IV may include the MAPK gene $j n k-1$ or the p38 homolog pmk-1, additionally supporting the role of these genes in the nematode's expression response. The enriched eQTLs from chromosome IV and the left arm of the X chromosome also lie within the QTLs identified to influence behavioral defense against $B$. thuringiensis (Fig. 2). Taken together, we conclude that clusters 5 and 6 comprise the components of a general defense response, apparently active not only against pathogens but also other stressors and mediated by central stress and immune response pathways. In the $n p r-1$ mutant, this defense response is strongly reduced towards both pathogens.

The specific response to $P$. aeruginosa is captured by two clusters (i.e., clusters 7 and 8; Fig. 9). They are enriched for eQTLs on chromosome I, V, and X (Table 2), although in 
chromosomal regions without any known regulator of immune defense. These clusters, especially the upregulated cluster, are however enriched for genes previously shown to respond to infection by the same pathogen (Fig. 10b) and also those involved in the response to oxidative stress and xenobiotics, including cadmium and pesticides (Fig. 10a, b). The response to oxidative stress and xenobiotics is dominated by an up-regulation of cytochrome P450 genes (Fig. 11b; Additional file 17). The up-regulated cluster is further influenced by the two main immunity pathways, the npr-1 gene, and also a GATA transcription factor (Figs. 10c, d). All of the latter components have previously been shown to be central for immune defense against $P$. aeruginosa, especially the p38 MAPK signalling cascade [65] and the GATA transcription factor ELT-2 [66]. The transcriptomic response to $P$. aeruginosa infection is additionally strongly influenced by cytochrome P450 expression, possibly as part of a general stress response to reduce oxidative stress (Fig. 10c). Because these responses are activated more strongly in the $\mathrm{N} 2$ strain, they are likely to mediate the observed higher resistance and behavioral defense for this strain compared to the npr-1(ur89) mutant (Figs. 6 and 7 and model in Fig. 12).

The specific response to $B$. thuringiensis is defined by four clusters, two up-regulated and two down-regulated groups of genes (i.e., clusters 1-4; Fig. 9). They are enriched for eQTLs in the middle of chromosome IV (Table 2), which could contain known defense regulators against $B$. thuringiensis, the MAPK genes $j n k-1$ and $p m k-1$ [67], and which lies within the QTL above identified to contribute to behavioral defense against this pathogen (Fig. 2). Enriched eQTLs are additionally found on the left arm of chromosome $\mathrm{V}$ and the right arm of the $\mathrm{X}$ chromosome (Table 2), in both cases without a link to any known immune regulator. Moreover, the upregulated clusters show an over-representation of genes involved in metabolic processes and phosphatase activity (Fig. 10a). They also include genes known to respond to the same pathogen and cadmium, as well as genes that are usually downrather than up-regulated upon osmotic and pesticide stress (Fig. 10b). These two upregulated clusters are enriched for an Ebox transcription factor binding motif and the corresponding targets (Fig. 10d). The two downregulated clusters show an enrichment for oxidation reduction and developmental genes (Fig. 10a), genes responsive to B. thuringiensis and cadmium (Fig. 10b), and genes controlled by insulin-like signalling, including glycoproteins (Fig. 10c). One of the down-regulated clusters also shows an enrichment of the GATA transcription factor targets (Fig. 10d).

Taken together, the results of our enrichment analyses allow us to propose a possible mechanistic basis for the contrasting defense effects of the npr-1 gene. It is worth

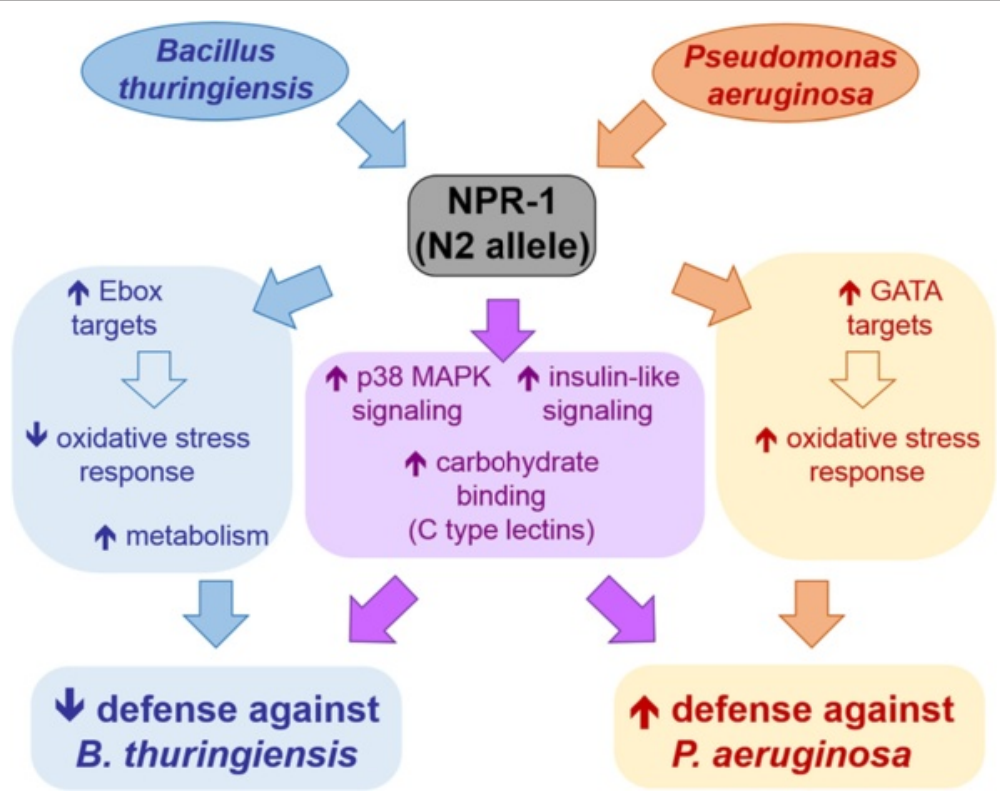

Fig. 12 Model of npr-1 dependent effects on pathogen defense in the N2 C. elegans strain. Exposure to both pathogens leads to an npr-1 dependent activation of carbohydrate-binding factors, such as C-type lectin-like proteins, and also two central immune signaling cascades, the p38 MAPK and the insulin-like pathways, which could all enhance pathogen resistance (middle part of the graph). Upon exposure to P. aeruginosa PA14 (right side), npr-1 also influences the activation of a general stress response, via one or several GATA transcription factors(s), which increases oxidative stress resistance and thus resistance to the pathogen. The response to $B$. thuringiensis (left side) is mediated by npr- 1 through one or several Ebox transcription factors, resulting in a reduced oxidative stress response and increased metabolic activity as a possible cause of enhanced pathogen susceptibility. Arrows with light colors indicate uncertain connections 
reiterating that $\mathrm{N} 2$ and the mutant only differ in the presence of a mutation in the npr-1 gene. Therefore, the differences observed between strains must be influenced by the allelic variation in this gene. The higher resistance and avoidance behavior of $\mathrm{N} 2$ towards $P$. aeruginosa is likely influenced by the activation of GATA and/or p38 MAPK targets and/or the induced oxidative stress response (see above; Fig. 12). The situation is less clear for the response to $B$. thuringiensis. Because N2 produces lower defenses against $B$. thuringiensis than the npr1(ur89) mutant (Figs. 4 and 5), and because any differential gene expression is repressed in the npr-1(ur89) mutant (Table 1; Fig. 9), the specific activation of certain genes in N2 (i.e., for the two up-regulated clusters 1 and 2) and/or the suppression of other gene groups in N2 (i.e., the down-regulated clusters 3 and 4) must account for the observed lower resistance and avoidance response against this pathogen. We hypothesize that this may possibly be mediated by one of the following two processes or a combination thereof (see model in Fig. 12): (i) the lower oxidative stress response in N2 could lead to increased susceptibility towards $B$. thuringiensis, in analogy to the effect recently described towards E. faecalis [68] and assuming that $B$. thuringiensis toxicity causes oxidative stress (which is however currently unknown); and/or (ii) an activation of metabolic processes could be disadvantageous during pathogen infection, because it may reduce availability of energetic resources that can be invested in immune defense and because metabolic products may be exploited as a source of nutrition by the pathogen. Any of the other implicated functions (Fig. 10) may also contribute to enhanced susceptibility in an as yet unknown manner. These processes then seem to be influenced by $n p r-1$ through Ebox-specific transcription factors. Interestingly, the higher susceptibility is apparently caused by an activation of specific functions and signalling processes rather than their absence or at least reduced activity. This may indicate a sub-optimal response to this specific pathogen in the N2 strain or represent a consequence of pathogen-mediated manipulation of host responses, which are widespread among pathogens [69] and which have also been shown for another Bacillus species, Bacillus nematocida, to change C. elegans behavior and intestinal responses [70]. At the moment, it is unclear in what way the indicated processes influence either behavioral or physiological responses or both simultaneously. This represents a challenging topic for future research.

\section{Conclusion}

Our study revealed a complex genetic architecture comprising several epistatically interacting QTLs associated with variation in $C$. elegans pathogen avoidance behavior. The most significant QTL encompassed the gene npr-1.
Our functional analyses of this gene revealed a contrasting effect of npr-1 on C. elegans immune defense, as assessed through both behavioral and also survival phenotypes. In particular, the CB4856 allele was associated with faster lawn leaving behavior and higher survival than the N2 allele on $B$. thuringiensis, whereas it was associated with lower lawn leaving behavior and lower survival on $P$. aeruginosa. A further characterization of the exact role of npr1 suggested that it mediates differential regulation of defense genes via either GATA transcription factors leading to increased immune defense towards $P$. aeruginosa or Ebox transcription factors leading to decreased immune defense towards $B$. thuringiensis. Our study thus demonstrates that a single gene in $C$. elegans mediates contrasting pathogen-specific defense responses.

\section{Methods}

\section{C. elegans and bacterial strains}

C. elegans strains: (i) the standard wild-type strains N2 and CB4856; (ii) 200 Recombinant Inbred Lines (RILs) and 90 Introgression lines (ILs) generated from crosses between N2 and CB4856 [41, 42, 55, 60]; and (iii) two distinct mutant alleles of $n p r-1, n p r-1$ (ur89) X (strain IM222) and $n p r-1$ (ad609) X (strain DA609), and also the tyra-3 knock-out allele tyra-3(ok325) X (strain VC125). The three mutant strains were obtained from the Caenorhabditis Genetics Center (CGC; http://www.cbs.umn.edu/CGC/) and were all generated in the N2 background. All worm strains were maintained at $20{ }^{\circ} \mathrm{C}$ on Nematode Growth Medium (NGM) plates with the non-pathogenic E. coli OP50 as an ad libitum food according to standard protocols [71]. All mutants were backcrossed at least three times to N2. Presence of the target mutation was confirmed for the two npr-1 mutants by sequencing the npr-1 gene at the location of the mutations and for the knockout mutant tyra-3 by polymerase chain reaction (PCR) analysis of the deleted region.

Bacterial strains: (i) two nematocidal strains of B. thuringiensis, NRRL B-18247 and NRRL B-18679, originally provided by the Agriculture Service Patent Culture Collection (United States Department of Agriculture, Peoria, Illinois, USA); (ii) the non-nematocidal $B$. thuringiensis strain DSM350, originally obtained from the German Collection of Microorganisms and Cell Cultures (Deutsche Sammlung von Mikroorganismen und Zellkulturen GmbH, DSMZ, Braunschweig, Germany); (iii) the pathogenic $P$. aeruginosa strain PA14, obtained from Dennis H. Kim, Boston, USA; and (iv) the non-pathogenic E. coli OP50. Before the start of this study, the three $B$. thuringiensis strains were cultured in large quantities as previously described [32]. The cultures consisted mainly of spores associated to nematocidal toxins in the case of B-18679 and B-18247, and non nematocidal toxins in the case of the control DSM 350. All cultures were set to a concentration of $1.5 \times 10^{10}$ particles $/ \mathrm{ml}$, 
assessed through standard Thoma counting chambers and microscopic analysis. The cultures were cryo-preserved in aliquots at $-20{ }^{\circ} \mathrm{C}$; spore viability and pathogenicity are not affected by freezing under these conditions [38, 40, 72]. Usage of viable spore aliquots from the same starting culture allowed us to minimize variation across experiments, thus enhancing comparability of the data from different study approaches. In all cases, aliquots were thawed directly before each experiment. The bacterial cultures were then diluted, as indicated below in the description of the various assays.

\section{Lawn leaving assays}

The assay was designed for $9 \mathrm{~cm}$ petri dishes with either Peptone free NGM (PFM) for B. thuringiensis assays or peptone-rich NGM for PA14 assays. $30 \mu \mathrm{l}$ of the test bacterium were spotted onto the center of the plate and $80 \mu \mathrm{l}$ of $E$. coli OP50 only were additionally placed in the shape of a ring (Additional file 1: Figure S1). This ring of OP50 protects escaping worms from starvation, minimizing their return to the lawn in the center. The test bacterium consisted of either $B$. thuringiensis diluted with OP50 at 1:250 from a stock with a concentration of $1.5 \times 10^{10}$ particles $/ \mathrm{ml}$, or PA14 diluted with OP50 in a 4:1 ratio. 10 hermaphroditic fourth instar larvae (L4) were picked onto the test lawn. Experiments were performed at $20^{\circ} \mathrm{C}$.

Leaving behavior was recorded by counting the number of worms on the lawn at different time points of exposure and calculated according to the following formula:

Leaving index $=\frac{10-\text { number of worms on the lawn }}{10}$

The screens of RILs (200 lines) and ILs (90 lines) were done using a randomized block design on 17 dates, always including the parental strains of these lines, N2 and CB4856, as internal controls. Each C. elegans strainbacteria-time point combination was assayed in three replicates, resulting in a total of 35040 individual data points. The screens of $n p r-1$ mutants included 12 replicates of each treatment.

We would like to note that the leaving assay for PA14 was performed at $20{ }^{\circ} \mathrm{C}$ and thus at a different temperature than the standard PA14 survival assays at $25^{\circ} \mathrm{C}$ (see below and [19]). The reason is that the $25{ }^{\circ} \mathrm{C}$ temperature led to increased bacterial growth on the assay plate, which caused enhanced dispersal of bacterial colonies through the crawling worms, thus compromising reliable scoring of the leaving behavior. Such a bias was not observed at $20^{\circ} \mathrm{C}$. As our results did confirm previously published data on C. elegans avoidance behavior towards PA14 [19], our assay conditions allowed us to characterize a robust behavioral response against this pathogen.

\section{Survival assays}

For survival analysis with $B$. thuringiensis, $6 \mathrm{~cm}$ peptone free NGM plates were inoculated with $100 \mu \mathrm{l}$ of a mixture of $B$. thuringiensis with $E$. coli OP50. Mixtures were prepared in seven dilutions: 1:2, 1:5, 1:10, 1:30, 1:50, 1:100, and 1:250 (equivalent to the relative concentration given in the main text). Plates were left to dry overnight $(9-15 \mathrm{~h})$ at $20{ }^{\circ} \mathrm{C} .30 \mathrm{~L} 4$ hermaphrodites were picked onto each assay plates. After $24 \mathrm{~h}$, survival was recorded by counting alive, dead and missing worms. Each treatment group was replicated 8 times across 8 runs (one replicate per run).

Analysis of $P$. aeruginosa effects was based on $3 \mathrm{~cm}$ peptone-rich NGM plates, which were inoculated with $5 \mu \mathrm{l}$ of an overnight culture at $37^{\circ} \mathrm{C}$ of either PA14 or OP50. Seeded plates were incubated overnight at $37{ }^{\circ} \mathrm{C}$ and then at $25{ }^{\circ} \mathrm{C} .30 \mathrm{~L} 4$ hermaphrodites were picked onto each assay plate and stored at $25{ }^{\circ} \mathrm{C}$ in the dark. Alive and dead worms were scored every $24 \mathrm{~h}$ and surviving worms were transferred to new assay plates every $48 \mathrm{~h}$. Each treatment group was replicated 10 times across two runs.

\section{Statistical analysis of phenotypic data}

We used the non-parametric Kruskall-Wallis test to assess differences in leaving behavior between the C. elegans strains, and a Bonferroni based adjustment to correct for multiple testing. We used Kaplan Meier analysis applying the Log Rank test to assess differences in C. elegans' survival on PA14. A Bonferroni based adjustment was used to correct for multiple testing. We used GLM ordinal logistic regression analysis to assess differences in survival between the $C$. elegans strains across the concentration range of $B$. thuringiensis, using C. elegans strains, BT concentration and the interaction between the two as factors. A Bonferroni based adjustment was used to correct for multiple testing. All escape and survival assays data were analyzed using the program JMP version 9.0 (SAS Institute Inc.), while graphic illustrations were produced with the program SIGMAPLOT version 12.0 (Systat Software Inc.).

\section{Quantitative trait locus (QTL) analysis}

The QTL analysis was performed on the average of three replicates per genotype/line of the calculated proportion "leave" (see assay method) of 200 Recombinant Inbred Lines (RILs) [41, 55-58, 73-76]. QTLs were calculated by single marker mapping using a linear model (trait marker + error) for each marker using a custom written script in the statistical programming language " $R$ " [77]. Significance levels were estimated from 1000 permutations 
of the data. The analysis calculated the significance of the linkage between the genetic marker and the trait one by one for each bacterium and exposure time point separately. We furthermore evaluated epistatic interactions between each two markers across the genome for each bacterium separately using the following model: phenotype $\sim$ time + marker1 " marker2. BIN mapping in the set of 90 ILs was done as described in $[42,56]$.

\section{RNA isolation and sequencing}

N2 and npr-1(ur89) worms were exposed to either PA14, BT B-18247 or OP50 for either 12 or $24 \mathrm{~h}$ of exposure. The experiment had 3 replicates of each treatment combination (a total of 36 samples across treatment combinations and replicates). Exposure experiments were performed on large Agar plates (15 cm diameter), which were fully covered with a bacterial lawn, thus minimizing escape responses and resulting in comparable occupancy rates across the treatment combinations. At both time points (12 \& 24), worms were washed off the exposure plates with PBS containing $0.3 \%$ Tween $20^{\circ}$ and resuspended in TRIzol $^{\circ}$ (Life Technologies) reagent. Prior to RNA extraction, worm suspensions were treated five times with a freeze-thaw cycle using liquid nitrogen and a thermo block at $45{ }^{\circ} \mathrm{C}$. RNA extraction was performed using a NucleoSpin ${ }^{\circ}$ mRNA extraction kit (Macherey-Nagel). RNA samples were treated with DNAse, and then stored at $-80^{\circ} \mathrm{C}$. RNA libraries were prepared for sequencing using standard Illumina protocols. Libraries were sequenced on an Illumina HiSeq ${ }^{\text {ma }} 2000$ sequencing machine with a paired-end strategy and read length of 100 nucleotides.

\section{Statistical analysis of transcriptomic data}

RNAseq reads were mapped to the C. elegans genome from Wormbase version WS235 (www.wormbase.org) by Tophat2 [78] using option -b2-very-sensitive, other default settings and without a transcriptome reference. Tophat 2 aligns RNAseq reads to a genome based on the ultra-fast short read mapping program Bowtie [79]. Estimation of transcript abundance and significantly differentially expressed genes were identified by Cuffdiff [80] using the quartile normalization method [81]. Cuffdiff is a program from the Cufflinks package and aims to find significant changes in transcript expression in consideration of possible formation of isoforms for a particular gene. The raw data is available from the GEO database $[82,83]$ under the GSE number GSE60063.

For clustering and visualization, transcripts with a significant change between different conditions (adjusted $p$ value $<0.01$ by the false discovery rate, FDR) were treated as signature for each comparison. Due to the biological variation of the replicates, the $p$-value, instead of foldchange, of those genes were firstly log10-transformed and ordered according to increasing or decreasing expression and then taken as input for k-means cluster analysis using cluster 3.0 [84] with a $\mathrm{k}$ of 8 . A heatmap was generated by TreeView version 1.1.4r3 [85]. Principal component analysis (PCA) was carried out on log-transformed gene expression profiles using a probabilistic PCA algorithm [86] from R package pcaMethods [87], which links PCA to the probability density of patterns. Dimensionality of the samples was reduced from 57165 (total isoforms) to three dimensions (PCs). Motif analysis was carried out on the promoter regions, $-600 \mathrm{bp}$ and $250 \mathrm{bp}$ relative to transcription start sites (TSS), of genes in each group. De novo motif discovery was performed by AMD [54].

\section{GO and EASE analysis}

Gene ontology (GO) and a gene set enrichment analysis was carried out on each group of genes from the $\mathrm{K}$ means cluster analysis. GO analysis was performed using DAVID $[52,53]$ with a cut-off of $p$-value $<0.05$, adjusted by FDR. For the gene set analysis, we used EASE [50], a free, stand-alone software package from DAVID bioinformatics resources (http://david.abcc.ncifcrf.gov/). As recently described [51, 64], we constructed a C. elegans-specific gene set database, WormExp [51], from published data and also using the previously established data sets collected by Ilka Engelmann et al., [88]. Based on this data set, we performed the EASE analysis and selected the results with a Bonferroni adjusted $p$-value $<0.05$.

\section{eQTL enrichment analysis}

eQTL enrichment was done using a hypergeometric test in R. The eQTL sets [41, 57-59] were obtained from WormQTL.org $[55,60]$. The eQTLs at a specific locus were compared to the genes in a specific expression cluster, as identified from the above described K-means cluster analysis.

\section{Ethics approval and consent to participate \\ Not applicable.}

\section{Consent for publication \\ Not applicable.}

\section{Availability of data and material}

The datasets, supporting the conclusions of this article, is available in case of the QTL analysis from WormQTL $[55,60]$, in case of the phenotypic analysis of N2, CB4856, and the $n p r-1$ mutants in Additional file 2 of this article, and in case of the transcriptome analysis from the GEO database [82, 83] under the GSE number GSE60063. The C. elegans strains are available from the Caenorhabditis Genetic Center (CGC), which is funded by NIH Office of Research Infrastructure Programs (P40 OD010440). All bacterial strains are available from the corresponding author upon request. 


\section{Additional files}

Additional file 1: Illustration of the lawn leaving assay. 10 hermaphrodites at the $L 4$ stage were transferred by picking onto $9 \mathrm{~cm}$ peptone free NGM plates containing a lawn of the tested bacteria, each mixed with E. coli OP50 and surrounded by a ring of $80 \mu \mathrm{l}$ of OP50. (TIF $144 \mathrm{~kb}$ )

Additional file 2: Raw data for the analysis of N2, CB4856, and the npr1 mutants. Sheet 1, Lawn leaving behavior of N2 and CB4856 from Bacillus thuringiensis - Raw data for Fig. 1; sheet 2, Lawn leaving behavior of mutants from Bacillus thuringiensis - Raw data for Fig. 4; sheet 3, Surival of N2 and CB4856 on Bacillus thuringiensis - Raw data for Additional file 9; sheet 4, Surival of mutants on Bacillus thuringiensis - Raw data for Fig. 5; sheet 5, Lawn leaving behavior of N2 and CB4856 from Pseudomonas aeruginosa PA14 - Raw data for Additional file 10; sheet 6, Lawn leaving behavior of mutants from Pseudomonas aeruginosa PA14 Raw data for Fig. 6; sheet 7, Surival of mutants on Pseudomonas PA14 - Raw data for Fig. 5. (XLS 482 kb)

Additional file 3: Table on the statistical results for the comparison between N2 and CB4856 leaving behavior towards B. thuringiensis and E. coli. (PDF $83 \mathrm{~kb}$ )

Additional file 4: Table on the statistical results for the pairwise comparisons of the leaving response on the E.coli OP50 control versus the $B$. thuringiensis strains. (PDF $87 \mathrm{~kb}$ )

Additional file 5: Figure on the leaving phenotypes of the introgression lines (ILs) plotted against the introgression position along the chromosomes. (A) Results for E. coli strain OP50; (B) non-nematocidal B. thuringiensis strain DSM350; (C) nematocidal B. thuringiensis B-18247; and (D) highly nematocidal B. thuringiensis B-18679. Green and red lines show the results after either $14 \mathrm{~h}$ or $24 \mathrm{~h}$ exposure, respectively. Position of markers is given along the $X$ axis. Light gray vertical lines indicate boundaries of the chromosomes. (TIF $1279 \mathrm{~kb}$ )

Additional file 6: Table on the statistical results for the comparison of the N2 and CB4856 leaving behavior with that of the mutant strains towards B. thuringiensis and E. coli. (PDF $96 \mathrm{~kb}$ )

Additional file 7: Table on the statistical results for the separate comparison between C. elegans N2 and CB4856 survival on nematocidal B. thuringiensis. (PDF $74 \mathrm{~kb}$ )

Additional file 8: Table on the statistical results for the pairwise comparison of survival of the $C$. elegans strains on nematocidal B. thuringiensis. (PDF $78 \mathrm{~kb}$ )

Additional file 9: Figure on the separate analysis of N2 and CB4856 survival in the presence of nematocidal $B$. thuringiensis. (A) Survival on $B$. thuringiensis strain B-18247; and (B) B-18679. Survival on the $Y$ axis is plotted against $\mathrm{BT}$ concentration on the $\mathrm{X}$ axis. Error bars represent standard error of the means. The statistical results are given in Additional file 6. (TIF $106 \mathrm{~kb}$ )

Additional file 10: Figure on the separate analysis of lawn leaving behavior of $\mathrm{N} 2$ and CB4856 towards E. coli and P. aeruginosa. (A) Results for avoidance of E. coli strain OP50; and (B) P. aeruginosa strain PA14. The asterisk ${ }^{*}$ ) points to a significant difference to N2. The dotted reference line indicates the 0.5 avoidance response. Statistical results are shown in Additional file 10. (TIF $97 \mathrm{~kb}$ )

Additional file 11: Table on the statistical results for the comparison between N2 and CB4856 leaving behavior towards E. coli and $P$. aeruginosa. (PDF $76 \mathrm{~kb}$ )

Additional file 12: Table on the statistical results for the comparison of the N2 and CB4856 leaving behavior with that of the mutant strains towards P. aeruginosa and E. coli. (PDF $90 \mathrm{~kb}$ )

Additional file 13: Table on the statistical results for the comparison of C. elegans survival rate on $P$. aeruginosa PA14 versus the control E. coli OP50. (PDF $74 \mathrm{~kb}$ )

Additional file 14: Table on the statistical results for the pairwise comparisons of $C$. elegans survival rates on $P$. aeruginosa PA14. (PDF 72 kb)

Additional file 15: Table with the list of differentially expressed genes after exposure of the $C$. elegans N2 wildtype or npr-1 mutant to pathogenic $B$. thuringiensis B-18247, pathogenic $P$. aeruginosa PA14, or non-pathogenic E. coli. (XLS 2096 kb)
Additional file 16: Results of the eOTL enrichment analysis of the $K$ means clusters of differentially expressed genes. (XLS $160 \mathrm{~kb}$ )

Additional file 17: Results of the GO term enrichment analysis (first sheet), the motif analysis (second sheet), and the C. elegans-specific EASE enrichment analysis (third sheet) of the $\mathrm{K}$ means clusters of differentially expressed genes. (XLS $316 \mathrm{~kb}$ )

\section{Abbreviations}

AMD: automated motif discovery; DAVID: database for annotation, visualization and integrated discovery; EASE: expression analysis systematic explorer; eQTL: expression quantitative trait locus; FDR: false discovery rate; GEO: gene expression omnibus; GLM: generalized linear model; GO: gene ontology; PCA: principal component analysis; QTL: quantitative trait locus.

\section{Competing interests}

The authors declare that they have no competing interests.

\section{Authors' contributions}

RN conceived the study, performed and participated in all experiments, analyzed the data, and drafted the manuscript. LBS conceived and performed QTL analysis and drafted the manuscript. WY performed transcriptomic analysis and drafted the manuscript. SE, FS, TGM, LR, ACM, KD helped performing phenotypic and functional genetic experiments. PCR conceived and participated in the transcriptomic analysis. JEK provided the RIL and IL libraries, conceived the QTL analysis, and drafted the manuscript. HS conceived the study, analyzed the data, and drafted the manuscript. All authors read and approved the final manuscript.

\section{Acknowledgements}

We are grateful to all members of the Schulenburg and Kammenga labs for support and advice. We thank particularly Christiana Anagnostou, Daniela Haase, Leila Masri, Barbara Pees, Joost Riksen, Stefanie Rohwer, and Anna Sheppard. We also thank the Kiel ICMB sequencing team (especially Markus Schilhabel, Melanie Friskovec, Melanie Schlapkohl) for technical assistance. The C. elegans strains were provided by the Caenorhabditis Genetic Center (CGC), which is funded by NIH Office of Research Infrastructure Programs (P40 OD010440). We thank the CGC for providing these strains and Dennis Kim for providing the $P$. aeruginosa strain PA14.

\section{Funding}

We are most grateful for financial support from the German Science Foundation to HS (DFG grants SCHU 1415/6 and SCHU 1415/9); Kiel University to HS and KD; infra-structural funds from the DFG Excellence Cluster Inflammation at Interfaces to HS and PR; the Netherlands Organisation for Scientific Research to LBS and JEK (grants 823.01.001 and 855.01.151); the HFSP to JEK; and the International Max Planck Research School (IMPRS) for Evolutionary Biology to WY.

\section{Author details}

Department of Evolutionary Ecology and Genetics, Zoological Institute University of Kiel, 24098 Kiel, Germany. ${ }^{2}$ Cologne Excellence Cluster for Cellular Stress Responses in Ageing-Associated Diseases (CECAD) and Systems Biology of Ageing, University of Cologne, Joseph-Stelzmann-Str. 26, 50931 Cologne, Germany. ${ }^{3}$ Laboratory of Nematology, Wageningen University, Wageningen 6708 PB, The Netherlands. ${ }^{4}$ Institute for Clinical Molecular Biology, University of Kiel, 24098 Kiel, Germany.

Received: 7 November 2015 Accepted: 25 March 2016 Published online: 11 April 2016

References

1. Schulenburg H, Boehnisch C, Michiels NK. How do invertebrates generate a highly specific innate immune response? Mol Immunol. 2007; 44(13):3338-44.

2. Irazoqui JE, Urbach JM, Ausubel FM. Evolution of host innate defence: insights from Caenorhabditis elegans and primitive invertebrates. Nat Rev Immunol. 2010;10(1):47-58.

3. Buchon N, Silverman N, Cherry S. Immunity in Drosophila melanogaster from microbial recognition to whole-organism physiology. Nat Rev Immunol. 2014;14(12):796-810. 
4. Meisel JD, Kim DH. Behavioral avoidance of pathogenic bacteria by Caenorhabditis elegans. Trends Immunol. 2014;35(10):465-70.

5. Ewbank JJ, Pujol N. Local and long-range activation of innate immunity by infection and damage in C. elegans. Curr Opin Immunol. 2016;38:1-7.

6. Luhachack LG, Visvikis O, Wollenberg AC, Lacy-Hulbert A, Stuart LM, Irazoqui JE. EGL-9 controls C. elegans host defense specificity through prolyl hydroxylation-dependent and-independent HIF-1 pathways. PLoS Pathog. 2012;8(7):e1002798.

7. Shao Z, Zhang Y, Ye Q, Saldanha JN, Powell-Coffman JA. C. elegans SWAN-1 Binds to EGL-9 and regulates HIF-1-mediated resistance to the bacterial pathogen Pseudomonas aeruginosa PAO1. PLoS Pathog. 2010;6(8): e1001075.

8. Bellier A, Chen C-S, Kao C-Y, Cinar HN, Aroian RV. Hypoxia and the hypoxic response pathway protect against pore-forming toxins in C. elegans. PLoS Pathog. 2009;5(12):e1000689.

9. Tenor $\mathrm{J}$, Aballay A. A conserved Toll-like receptor is required for Caenorhabditis elegans innate immunity. EMBO Rep. 2008;9(1):103-9.

10. Pujol N, Link EM, Liu LX, Kurz CL, Alloing G, Tan M-W, Ray KP, Solari R, Johnson CD, Ewbank JJ. A reverse genetic analysis of components of the Toll signaling pathway in Caenorhabditis elegans. Curr Biol. 2001; 11(11):809-21.

11. Schulenburg $H$, Ewbank JJ. The genetics of pathogen avoidance in Caenorhabditis elegans. Mol Microbiol. 2007;66(3):563-70.

12. Petersen $C$, Dirksen P, Schulenburg H. Why we need more ecology for genetic models such as C. elegans. Trends Genet. 2015;31:120-127.

13. Felix MA, Braendle C. The natural history of Caenorhabditis elegans. Curr Biol. 2010;20(22):R965-9.

14. Felix M-A, Duveau F. Population dynamics and habitat sharing of natural populations of Caenorhabditis elegans and C. briggsae. BMC Biol. 2012;10:59.

15. Petersen C, Dirksen P, Prahl S, Strathmann EA, Schulenburg H. The prevalence of Caenorhabditis elegans across 1.5 years in selected North German locations: the importance of substrate type, abiotic parameters, and Caenorhabditis competitors. BMC Ecol. 2014;14:4.

16. Pradel E, Zhang Y, Pujol N, Matsuyama T, Bargmann Cl, Ewbank JJ. Detection and avoidance of a natural product from the pathogenic bacterium Serratia marcescens by Caenorhabditis elegans. Proc Natl Acad Sci U S A. 2007;104(7):2295-300.

17. Reddy KC, Andersen EC, Kruglyak L, Kim DH. A polymorphism in npr-1 is a behavioral determinant of pathogen susceptibility in C. elegans. Science. 2009;323(5912):382-4.

18. Styer KL, Singh V, Macosko E, Steele SE, Bargmann Cl, Aballay A. Innate immunity in Caenorhabditis elegans is regulated by neurons expressing NPR1/GPCR. Science. 2008;322(5900):460-4.

19. Chang HC, Paek J, Kim DH. Natural polymorphisms in C. elegans HECW-1 E3 ligase affect pathogen avoidance behaviour. Nature. 2012;480(7378):525-9.

20. McMullan R, Anderson A, Nurrish S. Behavioral and immune responses to infection require Gaq- RhoA Signaling in C. elegans. PLoS Pathog. 2012;8(2): e1002530.

21. Hasshoff M, Boehnisch C, Tonn D, Hasert B, Schulenburg H. The role of Caenorhabditis elegans insulin-like signaling in the behavioral avoidance of pathogenic Bacillus thuringiensis. FASEB J. 2007;21(8):1801-12.

22. Zhang $Y, L u H$, Bargmann Cl. Pathogenic bacteria induce aversive olfactory learning in Caenorhabditis elegans. Nature. 2005;438(7065): 179-84.

23. Schulenburg H, Muller S. Natural variation in the response of Caenorhabditis elegans toward Bacillus thruingiensis. Parasitology. 2004;128:433-43.

24. Volkers RJM, Snoek LB, van Hellenberg Hubar CJ, Coopman R, Chen W, Yang W, Sterken MG, Schulenburg H, Braeckman BP, Kammenga JE. Geneenvironment and protein-degradation signatures characterize genomic and phenotypic diversity in wild Caenorhabditis elegans populations. BMC Biol. 2013;11(1):93.

25. Sicard M, Hering S, Schulte R, Gaudriault S, Schulenburg $H$. The effect of Photorhabdus luminescens (Enterobacteriaceae) on the survival, development, reproduction and behaviour of Caenorhabditis elegans (Nematoda: Rhabditidae). Environ Microbiol. 2007;9(1):12-25.

26. Glater $\mathrm{EE}$, Rockman MV, Bargmann Cl. Multigenic natural variation underlies Caenorhabditis elegans olfactory preference for the bacterial pathogen Serratia marcescens. G3. 2014;4(2):265-76.

27. Andersen EC, Gerke JP, Shapiro JA, Crissman JR, Ghosh R, Bloom JS, Felix M-A Kruglyak L. Chromosome-scale selective sweeps shape Caenorhabditis elegans genomic diversity. Nat Genet. 2012;44(3):285-90.
28. de Bono $\mathrm{M}$, Bargmann $\mathrm{Cl}$. Natural variation in a neuropeptide $\mathrm{Y}$ receptor homolog modifies social behavior and food response in C. elegans. Cell. 1998;94(5):679-89

29. Rogers C, Persson A, Cheung B, de Bono M. Behavioral motifs and neural pathways coordinating $\mathrm{O} 2$ responses and aggregation in C. elegans. Curr Biol. 2006;16(7):649-59.

30. Weber KP, De S, Kozarewa I, Turner DJ, Babu MM, de Bono M. Whole genome sequencing highlights genetic changes associated with laboratory domestication of C. elegans. PLoS One. 2010;5(11):e13922.

31. Bendesky A, Tsunozaki M, Rockman MV, Kruglyak L, Bargmann Cl. Catecholamine receptor polymorphisms affect decision-making in $\mathrm{C}$. elegans. Nature. 2012;472:313-318.

32. Borgonie G, Van Driessche R, Leyns F, Arnaut G, De Waele D, Coomans A. Germination of Bacillus thuringiensis spores in bacteriophagous nematodes (Nematoda: Rhabditida). J Invertebr Pathol. 1995:65(1):61-7.

33. Borgonie G, Claeys M, Leyns F, Arnaut G, De Waele D, Coomans AV. Effect of a nematicidal Bacillus thuringiensis strain on free-living nematodes. 3. Characterization of the intoxication process. Fundam Appl Nematol. 1996; 19:523-8.

34. Wei JZ, Hale K, Carta L, Platzer E, Wong C, Fang SC, Aroian RV. Bacillus thuringiensis crystal proteins that target nematodes. Proc Natl Acad Sci U S A. 2003;100(5):2760-5.

35. Griffitts JS, Aroian RV. Many roads to resistance: how invertebrates adapt to Bt toxins. Bioessays. 2005;27(6):614-24.

36. Nielsen-LeRoux C, Gaudriault S, Ramarao N, Lereclus D, Givaudan A. How the insect pathogen bacteria Bacillus thuringiensis and Xenorhabdus/ Photorhabdus occupy their hosts. Curr Opin Microbiol. 2012;15(3):220-31.

37. Wang J, Nakad R, Schulenburg H. Activation of the Caenorhabditis elegans FOXO family transcription factor DAF-16 by pathogenic Bacillus thuringiensis. Dev Comp Immunol. 2012;37(1):193-201.

38. Schulte RD, Makus C, Hasert B, Michiels NK, Schulenburg H. Host-parasite local adaptation after experimental coevolution of Caenorhabditis elegans and its microparasite Bacillus thuringiensis. Proc Royal Soc B. 2011;278(1719): 2832-9.

39. Schulte RD, Makus C, Hasert B, Michiels NK, Schulenburg H. Multiple reciprocal adaptations and rapid genetic change upon experimental coevolution of an animal host and its microbial parasite. Proc Natl Acad Sci. 2010;107(16):7359-64.

40. Masri L, Branca A, Sheppard AE, Papkou A, Laehnemann D, Guenther PS, Prahl S, Saebelfeld M, Hollensteiner J, Liesegang $H$. Host-pathogen coevolution: the selective advantage of Bacillus thuringiensis virulence and its cry toxin genes. PLoS Biol. 2015;13(6):e1002169.

41. Li Y, Ãlvarez OA, Gutteling EW, Tijsterman M, Fu J, Riksen JAG, Hazendonk E, Prins P, Plasterk RHA, Jansen RC, et al. Mapping determinants of gene expression plasticity by genetical genomics in C. elegans. PLoS Genet. 2006;2(12):e222.

42. Doroszuk A, Snoek LB, Fradin E, Riksen J, Kammenga J. A genome-wide library of CB4856/N2 introgression lines of Caenorhabditis elegans. Nucleic Acids Res. 2009;37(16):e110.

43. Balla KM, Troemel ER. Caenorhabditis elegans as a model for intracellular pathogen infection. Cell Microbiol. 2015;15(8):1313-22.

44. Wilfert L, Gadau J, Schmid-Hempel P. The genetic architecture of immune defense and reproduction in male Bombus terrestris bumblebees. Evolution. 2007:61(4):804-15.

45. Luijckx P, Fienberg H, Duneau D, Ebert D. A matching-allele model explains host resistance to parasites. Curr Biol. 2013;23(12):1085-8.

46. Schulenburg H, Kurtz J, Moret Y, Siva-Jothy MT. Introduction. Ecological immunology. Philo Transac Royal Soc B. 2009;364(1513):3-14.

47. Ferrandon D, Imler J-L, Hetru C, Hoffmann JA. The Drosophila systemic immune response: sensing and signalling during bacterial and fungal infections. Nat Rev Immunol. 2007;7(11):862-74.

48. Cheung BHH, Cohen M, Rogers C, Albayram O, de Bono M. Experiencedependent modulation of $\mathrm{C}$. elegans behavior by ambient oxygen. Curr Biol. 2005;15(10):905-17.

49. Chang AJ, Chronis N, Karow DS, Marletta MA, Bargmann Cl. A distributed chemosensory circuit for oxygen preference in C. elegans. PLoS Biol. 2006; 4(9):e274.

50. Hosack DA, Dennis Jr G, Sherman BT, Lane HC, Lempicki RA. Identifying biological themes within lists of genes with EASE. Genome Biol. 2003;4(10):R70.

51. Yang W, Dierking K, Schulenburg H. WormExp: a web-based application for a Caenorhabditis elegans-specific gene expression enrichment analysis. Bioinformatics. 2015; [Epub ahead of print]. 
52. Huang DW, Sherman BT, Lempicki RA. Systematic and integrative analysis of large gene lists using DAVID bioinformatics resources. Nat Protoc. 2008; 4(1):44-57.

53. Huang DW, Sherman BT, Lempicki RA. Bioinformatics enrichment tools: paths toward the comprehensive functional analysis of large gene lists. Nucleic Acids Res. 2009;37(1):1-13.

54. Shi J, Yang W, Chen M, Du Y, Zhang J, Wang K. AMD, an automated motif discovery tool using stepwise refinement of gapped consensuses. PLoS One. 2011;6(9):e24576

55. Snoek LB, Van der Velde KJ, Arends D, Li Y, Beyer A, Elvin M, Fisher J, Hajnal A, Hengartner MO, Poulin GB, et al. Worm QTL_public archive and analysis web portal for natural variation data in Caenorhabditis spp. Nucleic Acids Res. 2013;41(D1):D738-43.

56. Snoek LB, Orbidans HE, Stastna JJ, Aartse A, Rodriguez M, Riksen JAG, Kammenga JE, Harvey SC. Widespread genomic incompatibilities in Caenorhabditis elegans. G3. 2014;4(10):1813-23.

57. Li Y, Breitling R, Snoek LB, Velde KJ, Swertz MA, Riksen JAG, Jansen RC, Kammenga JE. Global genetic robustness of the alternative splicing machinery in Caenorhabditis elegans. Genetics. 2010;186:405-10.

58. Viñuela A, Snoek LB, Riksen JAG, Kammenga JE. Genome-wide gene expression regulation as a function of genotype and age in $C$. elegans. Genome Res. 2010;20(7):929-37.

59. Rockman MV, Skrovanek SS, Kruglyak L. Selection at linked sites shapes heritable phenotypic variation in C. elegans. Science. 2010; 330(6002):372-6.

60. Snoek LB, Joeri van der Velde K, Li Y, Jansen RC, Swertz MA, Kammenga JE. Worm variation made accessible: Take your shopping cart to store, link, and investigate! In: Worm. Abingdon, UK: Taylor \& Francis; 2014: e28357.

61. Miltsch SM, Seeberger PH, Lepenies B. The C-type lectin-like domain containing proteins CLEC-39 and CLEC-49 are crucial for Caenorhabditis elegans immunity against Serratia marcescens infection. Dev Compar Immunol. 2014;45(1):67-73.

62. Irazoqui JE, Troemel ER, Feinbaum RL, Luhachack LG, Cezairliyan BO, Ausubel FM. Distinct pathogenesis and host responses during infection of C. elegans by P. aeruginosa and S. aureus. PLoS Pathog. 2010;6(7):e1000982.

63. O'Rourke D, Baban D, Demidova M, Mott R, Hodgkin J. Genomic clusters, putative pathogen recognition molecules, and antimicrobial genes are induced by infection of C. elegans with M. nematophilum. Genome Res. 2006;16(8):1005-16.

64. Yang W, Dierking K, Esser D, Tholey A, Leippe M, Rosenstiel P, Schulenburg H. Overlapping and unique signatures in the proteomic and transcriptomic responses of the nematode Caenorhabditis elegans toward pathogenic Bacillus thuringiensis. Dev Compar Immunol. 2015;51(1):1-9.

65. Kim DH, Feinbaum R, Alloing G, Emerson FE, Garsin DA, Inoue H, Tanaka-Hino M, Hisamoto N, Matsumoto K, Tan M-W, et al. A conserved p38 MAP kinase pathway in Caenorhabditis elegans innate immunity. Science. 2002; 297(5581):623-6.

66. Shapira M, Hamlin BJ, Rong J, Chen K, Ronen M, Tan M-W. A conserved role for a GATA transcription factor in regulating epithelial innate immune responses. Proc Natl Acad Sci. 2006;103(38):14086-91.

67. Kao C-Y, Los FCO, Huffman DL, Wachi S, Kloft N, Husmann M, Karabrahimi V, Schwartz J-L, Bellier A, Ha C, et al. Global functional analyses of cellular responses to pore-forming toxins. PLoS Pathog. 2011;7(3):e1001314.

68. Feng N, Zhi D, Zhang L, Tian J, Ren H, Li C, Zhu H, Li H. Molecular mechanisms of resistance to human pathogenic bacteria in Caenorhabditis elegans by MEV-1 mediated oxidative stress. Biochem Biophys Res Commun 2015:459(3):481-7.

69. Schmid-Hempel P. Parasite immune evasion: a momentous molecular war. Trends Ecol Evol. 2008;23(6):318-26

70. Niu Q, Huang X, Zhang L, Xu J, Yang D, Wei K, Niu X, An Z, Bennett JW, Zou C. A Trojan horse mechanism of bacterial pathogenesis against nematodes. Proc Natl Acad Sci. 2010;107(38):16631-6.

71. Stiernagle T. Maintenance of C. elegans. WormBook. The C. elegans research community. In: WormBook. 2006.

72. Leyns F, Borgonie G, Arnaut G, De Waele D. Nematicidal activity of Bacillus thuringiensis isolates. Fundam Appl Nematol. 1995;18(3):211-8.

73. Gutteling EW, Doroszuk A, Riksen JAG, Prokop Z, Reszka J, Kammenga JE. Environmental influence on the genetic correlations between life-history traits in Caenorhabditis elegans. Heredity. 2007;98(4):206-13.

74. Viñuela A, Snoek LB, Riksen JAG, Kammenga JE. Aging uncouples heritability and expression-QTL in Caenorhabditis elegans. G3. 2012;2(5):597-605.
75. Elvin M, Snoek L, Frejno M, Klemstein U, Kammenga J, Poulin G. A fitness assay for comparing RNAi effects across multiple C. elegans genotypes. BMC Genomics. 2011;12(1):1-14.

76. Rodriguez M, Snoek LB, Riksen JAG, Bevers RP, Kammenga JE. Genetic variation for stress-response hormesis in C. elegans lifespan. Exp Gerontol. 2012:47(8):581-7.

77. R: A Language and Environment for Statistical Computing, R Core Team, R Foundation for Statistical Computing, Vienna, Austria, 2015, http://www.Rproject.org/. 2015

78. Kim D, Pertea G, Trapnell C, Pimentel H, Kelley R, Salzberg SL. TopHat2: accurate alignment of transcriptomes in the presence of insertions, deletions and gene fusions. Genome Biol. 2013;14(4):R36.

79. Langmead B, Trapnell C, Pop M, Salzberg SL. Ultrafast and memory-efficient alignment of short DNA sequences to the human genome. Genome Biol. 2009;10(3):R25.

80. Trapnell C, Hendrickson DG, Sauvageau M, Goff L, Rinn JL, Pachter L. Differential analysis of gene regulation at transcript resolution with RNA-seq Nat Biotechnol. 2013;31(1):46-53.

81. Robinson MD, Oshlack A. A scaling normalization method for differential expression analysis of RNA-seq data. Genome Biol. 2010;11(3):R25.

82. Edgar R, Domrachev M, Lash AE. Gene expression omnibus: NCBI gene expression and hybridization array data repository. Nucleic Acids Res. 2002; 30(1):207-10.

83. Barrett T, Wilhite SE, Ledoux P, Evangelista C, Kim IF, Tomashevsky M, Marshall KA, Phillippy KH, Sherman PM, Holko M. NCBI GEO: archive for functional genomics data sets update. Nucleic Acids Res. 2013;41(D1):D991-5.

84. de Hoon MJ, Imoto S, Nolan J, Miyano S. Open source clustering software. Bioinformatics. 2004;20(9):1453-4.

85. Saldanha AJ. Java Treeview-extensible visualization of microarray data. Bioinformatics. 2004;20(17):3246-8.

86. Tipping ME, Bishop CM. Probabilistic principal component analysis. J Royal Stat Soc Ser B. 1999:61(3):611-22.

87. Stacklies W, Redestig H, Scholz M, Walther D, Selbig J. pcaMethods-a bioconductor package providing PCA methods for incomplete data. Bioinformatics. 2007;23(9):1164-7

88. Engelmann I, Griffon A, Tichit L, Montanana-Sanchis F, Wang G, Reinke V, Waterston RH, Hillier LW, Ewbank JJ. A comprehensive analysis of gene expression changes provoked by bacterial and fungal infection in $\mathrm{C}$ elegans. PLoS One. 2011;6(5):e19055.

89. Huffman DL, Abrami L, Sasik R, Corbeil J, van der Goot FG, Aroian RV. Mitogen-activated protein kinase pathways defend against bacterial poreforming toxins. Proc Natl Acad Sci U S A. 2004;101(30):10995-1000.

90. Boehnisch C, Wong D, Habig M, Isermann K, Michiels NK, Roeder T, May RC, Schulenburg H. Protist-type lysozymes of the nematode Caenorhabditis elegans contribute to resistance against pathogenic Bacillus thuringiensis. PLoS One. 2011;6(9):e24619.

91. Troemel ER, Chu SW, Reinke V, Lee SS, Ausubel FM, Kim DH. p38 MAPK regulates expression of immune response genes and contributes to longevity in C. elegans. PLoS Genet. 2006;2(11):e183.

92. Park SK, Tedesco PM, Johnson TE. Oxidative stress and longevity in Caenorhabditis elegans as mediated by SKN-1. Aging Cell. 2009;8(3):258-69.

93. Rohlfing A-K, Miteva Y, Hannenhalli S, Lamitina T. Genetic and physiological activation of osmosensitive gene expression mimics transcriptional signatures of pathogen infection in C. elegans. PLoS One. 2010:5(2):e9010.

94. Cui Y, McBride SJ, Boyd WA, Alper S, Freedman JH. Toxicogenomic analysis of Caenorhabditis elegans reveals novel genes and pathways involved in the resistance to cadmium toxicity. Genome Biol. 2007;8(6):R122.

95. Murphy CT, McCarroll SA, Bargmann Cl, Fraser A, Kamath RS, Ahringer J, Li H, Kenyon C. Genes that act downstream of DAF-16 to influence the lifespan of Caenorhabditis elegans. Nature. 2003;424(6946):277-83.

96. McElwee JJ, Schuster E, Blanc E, Thomas JH, Gems D. Shared transcriptional signature in Caenorhabditis elegans Dauer larvae and long-lived daf-2 mutants implicates detoxification system in longevity assurance. J Bio Chem. 2004;279(43):44533-43.

97. Halaschek-Wiener J, Khattra JS, McKay S, Pouzyrev A, Stott JM, Yang GS, Holt RA, Jones SJM, Marra MA, Brooks-Wilson AR. Analysis of long-lived C. elegans daf-2 mutants using serial analysis of gene expression. Genome Res. 2005;15(5):603-15.

98. Kim SK, Lund J, Kiraly M, Duke K, Jiang M, Stuart JM, Eizinger A, Wylie BN, Davidson GS. A gene expression map for Caenorhabditis elegans. Science. 2001;293(5537):2087-92. 
99. Menzel R, Bogaert T, Achazi R. A systematic gene expression screen of Caenorhabditis elegans cytochrome P450 genes reveals CYP35 as strongly xenobiotic inducible. Arch Biochem Biophys. 2001;395(2):158-68.

100. Reichert K, Menzel R. Expression profiling of five different xenobiotics using a Caenorhabditis elegans whole genome microarray. Chemosphere. 2005; 61(2):229-37.

101. Grove CA, De Masi F, Barrasa MI, Newburger DE, Alkema MJ, Bulyk ML, Walhout AJM. A multiparameter network reveals extensive divergence between C. elegans bHLH transcription factors. Cell. 2009;138(2):314-27.

102. McGhee JD, Sleumer MC, Bilenky M, Wong K, McKay SJ, Goszczynski B, Tian H, Krich ND, Khattra J, Holt RA. The ELT-2 GATA-factor and the global regulation of transcription in the C. elegans intestine. Dev Biol. 2007;302(2):627-45.

103. Niu W, Lu ZJ, Zhong M, Sarov M, Murray Jl, Brdlik CM, Janette J, Chen C, Alves $P$, Preston $E$. Diverse transcription factor binding features revealed by genome-wide ChIP-seq in C. elegans. Genome Res. 2011;21(2):245-54.

Submit your next manuscript to BioMed Central and we will help you at every step:

- We accept pre-submission inquiries

- Our selector tool helps you to find the most relevant journal

- We provide round the clock customer support

- Convenient online submission

- Thorough peer review

- Inclusion in PubMed and all major indexing services

- Maximum visibility for your research

Submit your manuscript at www.biomedcentral.com/submit
Biomed Central 